\title{
NOVAS QUOTAS INFERIORES \\ PARA A PROBABILIDADE CRÍTICA DO PROCESSO DE PERCOLAÇÃO ORIENTADA POR MEIO DE CADEIAS DE MARKOV NUMA FAIXA DE MÚLTIPLAS VIAS
}

Thomas Logan Ritchie

\author{
DISSERTAÇÃO APRESENTADA \\ $\mathrm{AO}$ \\ INSTITUTO DE MATEMÁTICA E ESTATÍSTICA \\ DA \\ UNIVERSIDADE DE SÃO PAULO \\ PARA. \\ A OBTENÇÃO DO GRAU \\ DE \\ MESTRE EM ESTATÍSTICA \\ Área de Concentração: Probabilidade \\ Orientador: Prof. Dr. Vladimir Belitsky $\$$
}




\section{NOVAS QUOTAS INFERIORES \\ PARA A PROBABILIDADE CRÍTICA DO \\ PROCESSO DE PERCOLAÇÃO ORIENTADA \\ POR MEIO DE CADEIAS DE MARKOV \\ NUMA FAIXA DE MÚLTIPLAS VIAS}

Este exemplar corresponde à versão final da dissertação de mestrado de Thomas Logan Ritchie, corrigida e aprovada pela comissão julgadora.

São Paulo, 16 de março de 2001.

Comissão Julgadora:

- Prof. Dr. Vladimir Belitsky -IME-USP

- Prof. Dr. Mário José de Oliveira - IF-USP

- Prof ${ }^{a}$. Dra. Nancy Garcia Lopes - IMECC-UNICAMP 


\section{Agradecimentos}

Ao CNPQ pelo apoio financeiro e ao Professor Mikhail Menshikov por sua contribuição à formulação deste trabalho. 


\title{
Resumo:
}

Apresentamos uma seqüência decrescente de passeios aleatórios em $\mathbb{Z}$, que domina a margem direita do processo de percolação orientada. Através do conceito de cadeia de Markov numa faixa, descrevemos um algoritmo que gera uma seqüência crescente de quotas inferiores que converge para a probabilidade crítica do processo de percolação orientada em duas dimenssões. Calculando numericamente os dez primeiros termos dessa seqüência, obtivemos uma nova quota inferior para a probabilidade crítica deste processo: $p_{c} \geq 0,63328$.

\begin{abstract}
:
We present a coupled decreasing sequence of random walks on $\mathbb{Z}$ that dominates the edge process of oriented-bond percolation in two dimensions. Using the concept of Markov chain in a strip, we construct an algorithm that generates an increasing sequence of lower bounds that converges to the critical probability of oriented-bond percolation. Numerical calculations of the first ten lower bounds thereby generated lead to an improved,ie higher, lower bound to this critical probability, viz. $p_{c} \geq 0.63328$.
\end{abstract}




\section{Sumário}

1 Introdução 1

2 Definições e Construções 2

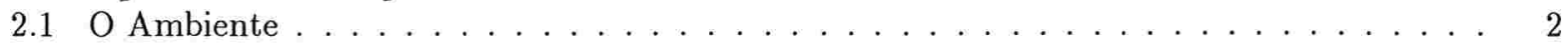

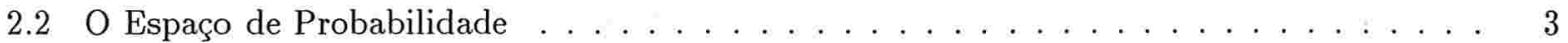

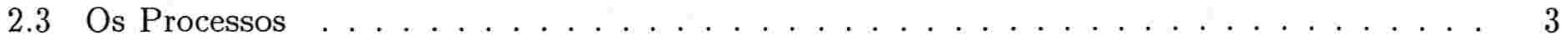

2.3.1 O Processo de Contato Fortalecido em Tempo Discreto (PCFTD) . . . . . . . . . 3

2.3.2 O Processo da Margem Direita (PMD) . . . . . . . . . . . . . . . . . . . . . . . 4

2.3.3 As Cadeias de Markov Induzidas . . . . . . . . . . . . . . . . . . . . . . . . . . . . .

2.4 Probabilidades Críticas . . . . . . . . . . . . . . . . . . . . 7

3 O Teorema de Convergência e Resultados Preliminares $\quad 8$

4 Determinação das Probabilidades Críticas $\quad 12$

4.1 Determinação Algébrica das Probabilidades Críticas . . . . . . . . . . . . . . . . . . 12

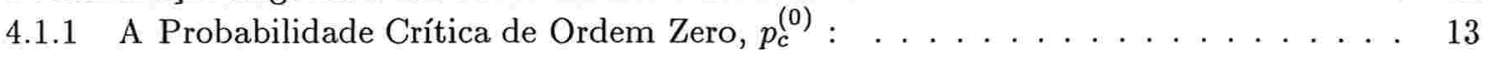

$4.1 .2 \quad$ A Probabilidade Crítica de Primeira Ordem, $p_{c}^{(1)}: \ldots \ldots \ldots$

4.1.3 A Probabilidade Crítica de Segunda Ordem, $p_{c}^{(2)}: \ldots \ldots \ldots$. . . . . . . . 14

4.2 Determinação Numérica das Probabilidades Críticas: . . . . . . . . . . . . . . . . . . 14

5 Simulações 17

5.1 Obtenção de Quotas Inferiores via Simulação . . . . . . . . . . . . . . . . . . . . 17

5.1 .1 Determinação de $p_{c}^{(5)} \ldots \ldots \ldots \ldots \ldots \ldots \ldots$

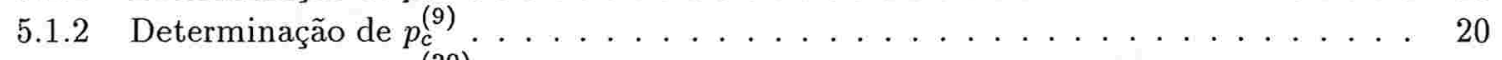

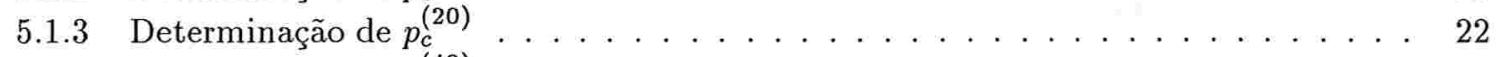

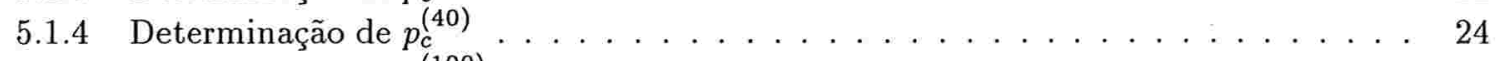

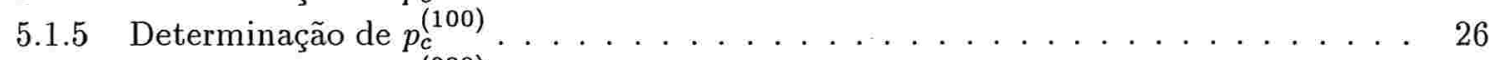

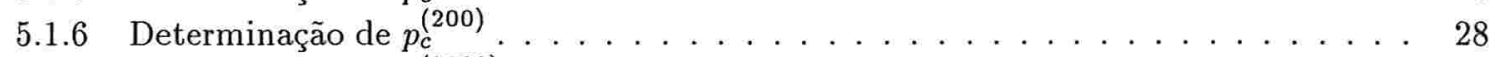

5.1 .7 Determinação de $p_{c}^{(1000)} \ldots \ldots \ldots \ldots \ldots \ldots \ldots \ldots$

5.2 Ilustração dos Acoplamentos via Simulação . . . . . . . . . . . . . . . . . . . . 32

$\begin{array}{ll}\text { A Programa I } & 35\end{array}$

$\begin{array}{lll}\text { B Programa II } & 46\end{array}$

\section{Lista de Tabelas}

1 Probabilidades críticas obtidas numericamente. . . . . . . . . . . . . . . . 16

2 Probabilidades críticas obtidas por simulação . . . . . . . . . . . . . . . . . . 17 


\section{Lista de Figuras}

$1 \quad \mathrm{O}$ grafo $\mathcal{G}$, onde os processos estudados serão definidos na Secção $2.3 \ldots \ldots \ldots \ldots$

2 Uma realização $\omega$ do PCFTD ${ }_{p} X_{\bullet}^{(2)}$. Os vetores em traço contínuo estão abertos, enquanto os vetores pontilhados estão fechados à propagação da infecção . Os sítios negros estão infectados, os brancos estão saudáveis, enquanto os hachureados foram infectados $\dot{a}$ força. . . . . . . . . (Refere-se ao lema 3.8) Não pode haver infecção à força dentro do paralelograma tracejado.

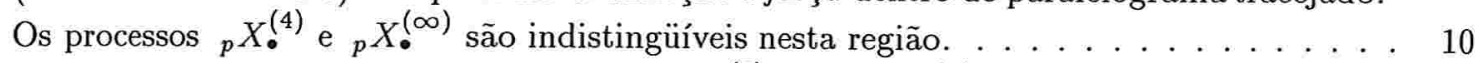

4 Realizações não acopladas dos processos ${ }_{.626} \bar{X}_{n}^{(5)} / n$ e ${ }_{6627} \bar{X}_{n}^{(5)} / n \ldots \ldots \ldots \ldots \ldots$

5 Ampliação e detalhe do gráfico na figura $4 \ldots \ldots \ldots \ldots \ldots \ldots$

6 Realizações não acopladas dos processos $.6332 \bar{X}_{n}^{(9)} / n$ e ${ }_{66333} \bar{X}_{n}^{(9)} / n \ldots \ldots \ldots \ldots \ldots .20$

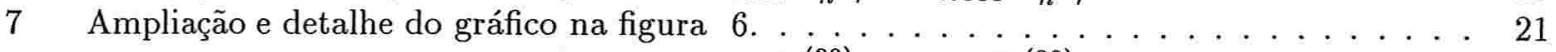

8 Realizações não acopladas dos processos ${ }_{.638} \bar{X}_{n}^{(20)} / n$ e ${ }_{.639} \bar{X}_{n}^{(20)} / n \ldots \ldots \ldots \ldots \ldots .22$

9 Ampliação e detalhe do gráfico na figura $8 \ldots \ldots \ldots \ldots \ldots \ldots \ldots$

10 Realizações não acopladas dos processos ${ }_{.641} \bar{X}_{n}^{(40)} / n$ e ${ }_{.642} \bar{X}_{n}^{(40)} / n \ldots \ldots \ldots \ldots \ldots .24$

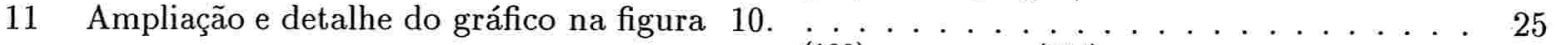

12 Realizações não acopladas dos processos ${ }_{.643} \bar{X}_{n}^{(100)} / n$ e ${ }_{.644} \bar{X}_{n}^{(100)} / n . \ldots \ldots \ldots \ldots . .26$

13 Ampliação e detalhe do gráfico na figura $12 \ldots \ldots \ldots \ldots \ldots \ldots$

14 Realizações não acopladas dos processos ${ }_{66438} \bar{X}_{n}^{(200)} / n$ e $.6439 \bar{X}_{n}^{(200)} / n$. . . . . . . . . . 28

15 Ampliação e detalhe do gráfico na figura $14 \ldots \ldots \ldots \ldots \ldots \ldots$

16 Realizações não acopladas dos processos $.64450 \bar{X}_{n}^{(1000)} / n$ e ${ }_{64452} \bar{X}_{n}^{(1000)} / n . \ldots \ldots \ldots . \quad 30$

17 Ampliação e detalhe do gráfico na figura $16 \ldots \ldots \ldots \ldots \ldots \ldots \ldots$

18 Realizações acopladas de ${ }_{6627} \bar{X}_{n}^{(3)} / n,{ }_{.627} \bar{X}_{n}^{(5)} / n$, e ${ }_{.627} \bar{X}_{n}^{(7)} / n \ldots \ldots \ldots \ldots \ldots \ldots .32$

19 Realizações acopladas de ${ }_{6629} \bar{X}_{n}^{(6)} / n, .630 \bar{X}_{n}^{(6)} / n$, e $.631 \bar{X}_{n}^{(6)} / n . \ldots \ldots \ldots \ldots \ldots$

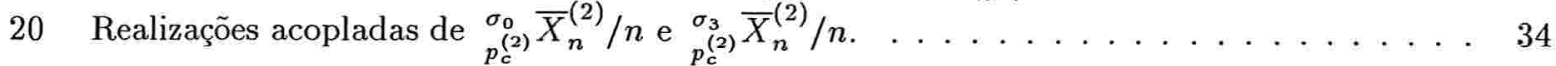




\section{Introdução}

Percolação orientada em duas dimensões, ou processo de contacto em tempo discreto em $\mathbb{Z}$, é uma família uniparamétrica de processos estocásticos com valores em $\{0,1\}^{\mathbb{Z}}$; onde o parâmetro $p$, a probabilidade de propagação local de infecção, assume valores em $[0,1]$. Sabe-se que esta família apresenta uma transição de fase, quando o valor de $p$ varia de 0 a 1 : se $p<p_{c}{ }^{1}$, o processo se extingüe quase certamente; enquanto que, se $p>p_{c}$, o processo sobrevive com probabilidade positiva (veja [1] por exemplo ).

Como é comum na teoria de fenômenos críticos, não se conhece uma expressão analítica para $p_{c}$. Desta forma o seu valor tem sido estimado tanto na literatura física, como na literatura matemática. $[1$, Sec.6]

Até o presente momento, as melhores estimativas rigorosas ${ }^{2}$ inferior e superior para $p_{c}$ são respectivamente 0,6298 e $2 / 3$. [2]

Neste trabalho apresentamos um algoritmo que gera uma seqüência crescente de quotas inferiores que converge para a probabilidade crítica do processo de percolação orientada em duas dimensões. Calculando os dez primeiros termos desta seqüência, pudemos estabelecer o valor de 0,63328 como quota inferior para $p_{c}$, o qual representa uma melhora em relação ao valor de 0,6298 .

Mais especificamente:

1. Construiu-se uma seqüência numérica de quotas inferiores para $p_{c},\left\{p_{c}^{(i)}\right\}_{i \in \mathbb{N}}$, nos seguintes passos:

(a) a partir de uma seqüência decrescente ${ }^{3}$ (em $i$ ) de passeios aleatórios em $\mathbb{Z},\left\{\bar{X}_{n}^{(i)}\right\}_{n \in \mathbb{N}}$, que dominam o processo da margem direita da percolação orientada, construiu-se uma seqüência (em $i$ ) de cadeias de Markov finitas $\left\{Y_{n}^{(i)}\right\}_{n \in \mathbb{N}}$, cujas probabilidades de transição são expressas em termos de " $p$ " 4 , a probabilidade de propagação local de infecção e

(b) calculou-se a esperança matemática da variável aleatória $\mathbb{E}\left(\bar{X}_{1}^{(i)}-\bar{X}_{0}^{(i)} \mid Y_{0}^{(i)}\right)$, "o salto médio na configuração $Y^{\prime \prime}$, com respeito a $\pi^{(i)}$, a medida estacionária de $Y_{\bullet}^{(i)}$.

(c) $p_{c}^{(i)}$ foi então definida como o único valor de $p$ que anula a esperança matemática acima. Em outras palavras, quando $p=p_{c}^{(i)}$, o passeio aleatório $\left\{\bar{X}_{n}^{(i)}\right\}_{n \in \mathbb{N}}$ não apresenta viés.

2. Mostrou-se que a seqüência numérica $\left\{p_{c}^{(i)}\right\}_{i \in \mathbb{N}}$ converge de modo não decrescente para $p_{c}$, ie $p_{c}^{(i)} \nearrow p_{c}$. Além disso,

3. as dez primeiras quotas inferiores $\left(p_{c}^{(0)}, p_{c}^{(1)}, \ldots, p_{c}^{(9)}\right)$ foram calculadas numericamente; o que possibilitou aumentar o valor de 0,6298 para 0,63328 , como melhor quota inferior para $p_{c}$, a probabilidade crítica para o proceso de percolação orientada em duas dimensões.

\footnotetext{
1 " $p_{c}$ " é conhecida como probabilidade crítica do processo de percolação orientada.

${ }^{2}$ obtidas por métodos matemáticos exatos.

${ }^{3}$ o significado exato de "decrescente" ficará claro em termos de acoplamento. (Secção 2)

${ }^{4}$ polinômios em $p$, mais precisamente.
} 


\section{Definições e Construções}

\subsection{O Ambiente}

Seja $\mathcal{G} \equiv(\mathcal{V}, \mathcal{E})$ o grafo orientado, tendo $\mathcal{V}=\{(n, m): n \in \mathbb{N}$ e $(n+m)$ seja par $\}$ como conjunto de vértices, e $\mathcal{E}=\left\{e_{n m}^{l}, e_{n m}^{r}:(n, m) \in \mathcal{V}\right\}$ como conjunto de elos.

O elo $e_{n, m}^{l}$ aponta do sítio $(n, m)$ para o sítio $(n+1, m-1)$, enquanto o elo $e_{n, m}^{r}$ aponta do sítio $(n, m)$ para o sítio $(n+1, m+1)$ (Figura 1 abaixo). Algumas vezes, faremos a associação natural : "l $\leftrightarrow-1 / r \leftrightarrow+1$ " ao longo deste texto.

É útil interpretar " $n$ " como sendo uma coordenada temporal e " $m$ " como uma coordenada espacial do grafo $\mathcal{G}$.

$\mathcal{V}_{n}$ denotará a $n^{\text {ésima }}$ fatia de $\mathcal{V}$, ie $\mathcal{V}_{n}=\{(n, m) \in \mathcal{V}: n$ esteja fixo $\}$, enquanto $\mathbb{Z}_{n}$ o conjunto dos números inteiros $m$ tais que $(m+n)$ seja par; ie $\mathbb{Z}_{n}$ é o conjunto dos inteiros de mesma paridade de $n$.

Nas definições abaixo identificaremos $\mathcal{V}_{n} \operatorname{com} \mathbb{Z}_{n}$, e $\{0,1\}^{\mathcal{V}_{n}} \operatorname{com}\{0,1\}^{\mathbb{Z}_{n}}$. Desta forma, o ligeiro abuso de notação : “ $\eta_{n}(m) \equiv \eta_{n}(n, m), \eta_{n} \in\{0,1\}^{\mathcal{V}_{n}}$ ”, deverá ser levado em conta.

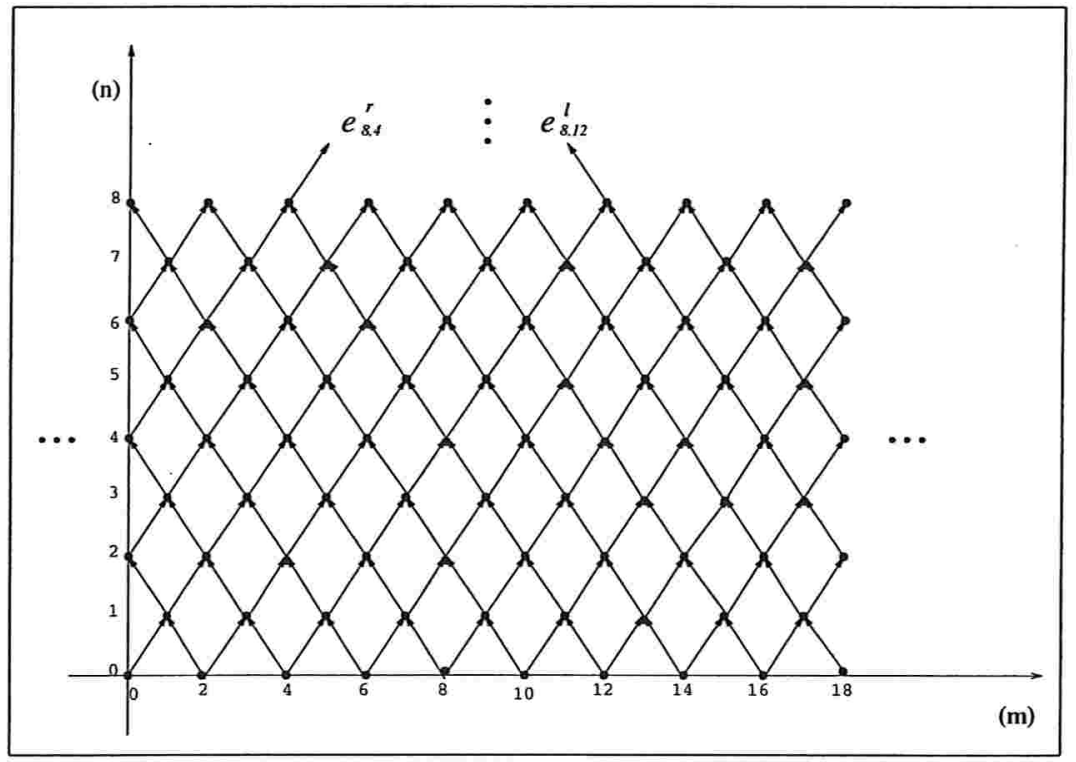

Figura 1: $O$ grafo $\mathcal{G}$, onde os processos estudados serão definidos na Secção 2.3 . 


\subsection{O Espaço de Probabilidade}

Seja $\left\{\xi_{n m}^{j}:(n, m) \in \mathcal{V}, j \in\{l, r\}\right\}$ uma família de variáveis aleatórias independentes e uniformemente distribuídas no intervalo $[0,1]$, definidas no mesmo espaço de probabilidade abstrato $\Omega$. A partir desta família enumerável e indexada por três índices, construímos, no mesmo espaço $\Omega$, a família (não -enumerável e indexada por quatro índices) de variáveis aleatórias de Bernoulli iids (para $p$ fixo):

$$
\left\{\xi_{n, m}^{j}:(n, m) \in \mathcal{V}, j \in\{l, r\}, p \in[0,1]\right\}
$$

tais que

$$
{ }_{p} \xi_{n, m}^{j}=\mathbf{1}_{\left\{\xi_{n m}^{j} \leq p\right\}}
$$

Segue diretamente de (2) que

$$
\left\{\begin{array}{l}
\mathbb{P}\left({ }_{p} \xi_{n m}^{j}=1\right)=p \\
\mathbb{P}\left({ }_{p}^{j} \xi_{n m}^{j}=0\right)=1-p:=q \quad(b)
\end{array}\right.
$$

, assim como o Acoplamento Fundamental:

$$
p_{1} \leq p_{2} \Rightarrow p_{1} \xi_{n m}^{j}(\omega) \leq p_{2} \xi_{n m}^{j}(\omega), \forall \omega \in \Omega,(n, m) \in \mathcal{V}, j \in\{l, r\}
$$

Observamos que ${ }_{p} \xi_{n m}^{l}=1$, pode ser interpretado como um canal aberto à propagação de uma infecção do sítio $(n, m)$ ao sítio $(n+1, m-1) ; p \xi_{n m}^{r}=0$, como um canal obstruído à propagação da infecção do sítio $(n, m)$ ao $(n+1, m+1)$; e assim em diante. .

\subsection{Os Processos}

\subsubsection{O Processo de Contato Fortalecido em Tempo Discreto (PCFTD)}

A configuração $\eta_{n} \in\{0,1\}^{\mathcal{V}_{n}}$, é interpretada como o estado de uma infecção na fatia $\mathcal{V}_{n}$ :

$$
\left\{\begin{array}{l}
\eta_{n}(m)=1: \text { o sítio } m \text { está infectado no instante } n \\
\eta_{n}(m)=0 ; \text { o sítio } m \text { está saudável no instante } n
\end{array}\right.
$$

Particularmente, $\eta_{0} \in\{0,1\}^{\mathcal{V}_{0}}$ denotará um estado inicial da infecção sobre $\mathcal{V}_{0}$; o conjunto dos números inteiros pares, de acordo com a secção 2.1 acima.

Neste ponto, chamamos atenção para a seguinte notação, que será utilizada no decorrer deste texto: $\mathbb{N}=\{0,1,2, \cdots\}$ e $\overline{\mathbb{N}}=\mathbb{N} \cup\{\infty\}$.

Agora, para cada $i \in \overline{\mathbb{N}}$, definimos, por indução sobre $n$, a sequência de variáveis aleatórias $\left\{{ }_{p} X_{n}^{(i)}\right\}_{n \in \mathbb{N}}$, assumindo valores em $\{0,1\}^{\mathcal{V}_{n}}$, pela

\section{Definição 2.1 .}

(a) ${ }_{p} X_{0}^{(i)}:=\eta_{0}$, de modo que ${ }_{p} X_{0}^{(i)}(m)=\eta_{0}(m), \forall m \in \mathbb{Z}_{0}$

(b) ${ }_{p} X_{n+1}^{(i)}(m)=\sup \left\{{ }_{p} X_{n}^{(i)}(m-1) \cdot{ }_{p} \xi_{n, m-1}^{r} ;{ }_{p} X_{n}^{(i)}(m+1) \cdot{ }_{p} \xi_{n, m+1}^{l} ; 1_{\left\{{ }_{p} \bar{X}_{n+1}^{(i)}-m>2 i\right\}}\right\}$, onde ${ }_{p} \bar{X}_{n+1}^{(i)}=\sup _{m \in \mathbb{Z}_{n} ; j \in\{-1,+1\}}\left\{(m+j):{ }_{p} X_{n}^{(i)}(m) \cdot \xi_{n m}^{j}=1\right\}$ 
O papel da função indicadora na definição 2.1(b) acima é infectar à força todos os sítios à esquerda e a uma distância superior a $2 i$ unidades de ${ }_{p} \bar{X}_{n+1}^{(i)}$, o sítio infectado mais à direita no instante $n+1$. É natural, portanto, denominar o processo estocástico $\left\{{ }_{p} X_{n}^{(i)}\right\}_{n \in \mathbb{N}}$, definido acima, como Processo de Contacto Fortalecido em Tempo Discreto (PCFTD) ( de iésima ordem e parâmetro de infecção $p$ ). Segue diretamente da definição 2.1 que

(a) quando $i=\infty$, a função indicadora não atua mais, e recupera-se o Processo de Contacto em Tempo Discreto habitual, também conhecido como Percolação Orientada em Duas Dimensões, como descrito em [1, Seç̧ão 2]. De agora em diante, nos referiremos a este processo como $\left\{{ }_{p} X_{n}^{(\infty)}\right\}_{n \in \mathbb{N}}$;

(b) a seqüência de PCFTDs $\left\{{ }_{p} X_{n}^{(i)}\right\}_{n \in \mathbb{N}}$ é decrescente em $i$, no seguinte sentido:

$$
i \leq j ; i, j \in \overline{\mathbb{N}} \Rightarrow{ }_{p} X_{n}^{(i)}(m)[\omega] \geq{ }_{p} X_{n}^{(j)}(m)[\omega], \forall \omega \in \Omega,(n, m) \in \mathcal{V}, p \in[0,1]
$$

Em particular, o Processo de Percolação Orientada é o mais fraco de todos eles.

Denominaremos a desigualdade 6 acima como Acoplamento de Primeira Ordem ou Acoplamento em $i$.

(c) a família de PCFTDs $\left\{{ }_{p} X_{n}^{(i)}\right\}_{n \in \mathbb{N}}$ é crescente em $p$, no seguinte sentido:

$$
p_{1} \leq p_{2} \Rightarrow{ }_{p_{1}} X_{n}^{(i)}(m)[\omega] \leq p_{2} X_{n}^{(i)}(m)[\omega], \forall \omega \in \Omega,(n, m) \in \mathcal{V}, i \in \overline{\mathbb{N}}
$$

Denominaremos a desigualdade 7 acima como Acoplamento de Segunda Ordem ou Acoplamento em $p$.

Salvo menção em contrário, assumiremos $\eta_{0}$, o estado inicial de infecção, como sendo a configuração $\mathbf{1}_{\bullet} \leq 0 \in\{0,1\}^{\mathcal{V}_{0}}$, dada por

$$
\mathbf{1}_{\bullet} \leq 0(m)=\left\{\begin{array}{l}
1, m \leq 0 \\
0, m>0
\end{array}\right.
$$

Quando for o caso, escreveremos $\left\{{ }_{p}^{\eta} X_{n}^{(i)}\right\}_{n \in \mathbb{N}}$ para enfatizar que $\eta_{0}=\eta$, onde $\eta$ representa alguma configuração em $\{0,1\}^{\nu_{0}}$ (provavelmente distinta de $\mathbf{1}_{\bullet} \leq 0$ ). Desta forma, todos os símbolos $\left\{{ }_{p} X_{n}^{(i)}\right\}_{n \in \mathbb{N}}$, $\left\{{ }^{\left.1 \cdot \leq_{p} X_{n}^{(i)}\right\}_{n \in \mathbb{N}},{ }^{1} \leq_{p} X_{\bullet}^{(i)},{ }_{p} X_{\bullet}^{(i)}}\right.$ assumem o mesmo significado e, por simplicidade, optaremos sempre pelo último, desde que não incorramos em ambigüidades .

Como é usual em Sistemas de Partículas Interagentes, escreveremos $\eta_{n} \geq \theta_{n}$, quando $\eta_{n}(m) \geq \theta_{n}(m)$, para todo $m \in \mathbb{Z}_{n}$.

Também segue da definição 2.1 que, para $\eta, \theta \in\{0,1\}^{\mathcal{V}_{0}}$

$$
\eta \geq \theta \Rightarrow{ }_{p}^{\eta} X_{n}^{(i)}(m)[\omega] \geq{ }_{p}^{\theta} X_{n}^{(i)}(m)[\omega], \forall p \in[0,1],(n, m) \in \mathcal{V}, \omega \in \Omega, i \in \overline{\mathbb{N}}
$$

Denominaremos a desigualdade 9 acima como Acoplamento de Terceira Ordem ou Acoplamento na Condição Inicial.

\subsubsection{O Processo da Margem Direita (PMD)}

Para um particular PCFTD ${ }_{p} X_{\bullet}^{(i)}$ e assumindo que ${ }_{p} \bar{X}_{0}^{(i)} \stackrel{\text { def }}{=} 0$, a segunda linha da definição 2.1(b) acima define um processo não markoviano em $\mathbb{Z}$ que representaremos por $\left\{{ }_{p} \bar{X}_{n}^{(i)}\right\}_{n \in \mathbb{N}}$ ( ou por ${ }_{p} \bar{X}_{\bullet}^{(i)} \mathrm{em}$ 


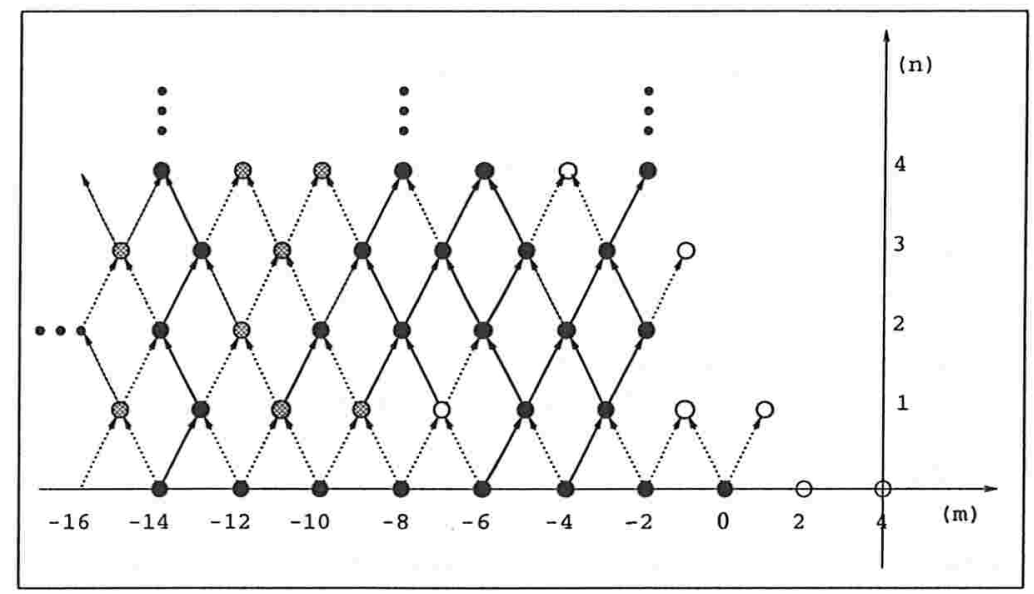

Figura 2: Uma realização $\omega$ do PCFTD ${ }_{p} X_{\bullet}^{(2)}$. Os vetores em traço contínuo estão abertos, enquanto os vetores pontilhados estão fechados à propagação da infeç̧ão. Os sítios negros estão infectados, os brancos estão saudáveis, enquanto os hachureados foram infectados à força.

forma abreviada).

Na figura 2 abaixo ${ }_{p} \bar{X}_{0}^{(2)}=0,{ }_{p} \bar{X}_{1}^{(2)}=-3,{ }_{p} \bar{X}_{2}^{(2)}=-2,{ }_{p} \bar{X}_{3}^{(2)}=-3,{ }_{p} \bar{X}_{4}^{(2)}=-2, \ldots$

Novamente, quando $i=\infty$, o Processo da Margem Direita da Percolação Orientada descrito em [1] é recuperado. Ao longo deste texto, o termo "Processo da Margem Direita" será utilizado lato sensu, ie para $i \in \overline{\mathbb{N}}$.

É interessante interpretar o PMD ${ }_{p} \bar{X}_{\bullet}^{(i)}$ como um passeio aleatório em $\mathbb{Z}$.

\subsubsection{As Cadeias de Markov Induzidas}

Definição 2.2 A cadeia de Markov $\left\{{ }_{p} Y_{n}^{(i)}\right\}_{n \in \mathbb{N}}$ com espaço de estados $\mathcal{S}^{(i)}=\{0,1\}^{\{2,4, \ldots, 2 i\}}$ definida por

$$
{ }_{p} Y_{n}^{(i)}(2 j)={ }_{p} X_{n}^{(i)}\left({ }_{p} \bar{X}_{n}^{(i)}-2 j\right), 1 \leq j \leq i
$$

chama-se Cadeia de Markov Induzida(CMI) pelo PCFTD ${ }_{p} X_{\bullet}^{(i)}$.

No que segue, uma configuração genérica $\sigma \in \mathcal{S}^{(i)}$, será indexada por $n \in\left\{0,1, \ldots, 2^{i}-1\right\}$ de acordo com a equação

$$
\sigma=\sigma_{n} \Leftrightarrow n=\sum_{j=1}^{i} 2^{i-j} \sigma(2 j)
$$

Desta forma, na figura 2 acima ${ }_{p} Y_{0}^{(2)}=\sigma_{3},{ }_{p} Y_{1}^{(2)}=\sigma_{2},{ }_{p} Y_{2}^{(2)}=\sigma_{3},{ }_{p} Y_{3}^{(2)}=\sigma_{3},{ }_{p} Y_{4}^{(2)}=\sigma_{1}, \ldots$ 
As probabilidades de transição para a CMI ${ }_{p} Y_{\bullet}^{(i)}$

$$
q_{l m}^{(i)}=\mathbb{P}\left[{ }_{p} Y_{n+1}^{(i)}=\sigma_{m} \mid{ }_{p} Y_{n}^{(i)}=\sigma_{l}\right], 0 \leq l, m \leq 2^{i}-1
$$

são funções polinomiais em $p$ estritamente positivas no intervalo $(0,1)$. Logo $\pi^{(i)}$, sua medida estacionária em $\mathcal{S}^{(i)}$, está bem definida.

A notação abreviada

$$
\pi_{l}^{(i)} \stackrel{\text { def }}{=} \pi^{(i)}\left(\sigma_{l}\right), l=0,1, \ldots, 2^{i}-1
$$

será empregada nas definições que seguem.

Neste ponto, de acordo com [3, Sec.3.1], estabelecemos as seguintes definições :

\section{Definição 2.3}

$$
P_{(l, m, k)}^{(i)}(p) \stackrel{\text { def }}{=} \mathbb{P}\left[{ }_{p} \bar{X}_{n+1}^{(i)}={ }_{p} \bar{X}_{n}^{(i)}+(1-2 k),{ }_{p} Y_{n+1}^{(i)}=\sigma_{m} \mid{ }_{p} Y_{n}^{(i)}=\sigma_{l}\right], k \in \mathbb{N}, 0 \leq l, m \leq 2^{i}-1
$$

as probabilidades de transição do estado $\sigma_{l}$ para o estado $\sigma_{m}$ com um salto de magnitude $(1-2 k)$.

Definição 2.4

$$
M_{l}^{(i)}(p) \stackrel{\text { def }}{=} \sum_{k=0}^{\infty} \sum_{m=0}^{2^{i}-1}(1-2 k) \cdot P_{(l, m, k)}^{(i)}(p), \quad 0 \leq l \leq 2^{i}-1
$$

o salto médio do PMD ${ }_{p} \bar{X}_{\bullet}^{(i)}$ na configuração $\sigma_{l}$.

Definição 2.5

$$
M^{(i)}(p) \stackrel{\text { def }}{=} \sum_{l=0}^{2^{i}-1} M_{l}^{(i)}(p) . \pi_{l}^{(i)}
$$

o salto médio ou "mean drift" do PMD ${ }_{p} \bar{X}_{\bullet}^{(i)}$.

$M^{(i)}(p)$ também será chamado de velocidade (média) da margem direita de acordo com a secção 2.4 abaixo. Como logo se fará claro, a Definição 2.5 é de importância fundamental neste trabalho.

$\grave{A}$ luz destas definições, os PCFTDs ${ }_{p} X_{\bullet}^{(i)}$, descritos acima, podem ser vistos, quando $i \in \mathbb{N}$, como Cadeias de Markov numa faixa de $2^{i}$ vias:

$$
{ }_{p} X_{n}^{(i)}={ }_{p} X_{n}^{(i)}\left({ }_{p} \bar{X}_{n}^{(i)},{ }_{p} Y_{n}^{(i)}\right)
$$

uma idéia ligeiramente diferente do conceito de Cadeias de Markov numa Meia Faixa, apresentado em $[3$, Sec.3.1]. 


\subsection{Probabilidades Críticas}

Na secção 3 , mostraremos que para $i \in \mathbb{N}$ e $p \in(0,1]$ :

(i) $M^{(i)}(p)$ é uma função estritamente crescente em $p$;

(ii) $M^{(i)}(p)$ tem uma única raiz real no intervalo $(0,1]$;

(iii) $\frac{p \bar{X}_{n}^{(i)}}{n} \stackrel{(n)}{\longrightarrow} M^{(i)}(p) \quad q s$.

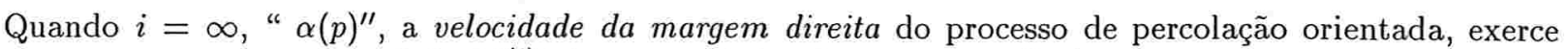
exatamente o mesmo papel de $M^{(i)}(p)$ no caso finito descrito acima ${ }^{5}$. (Para maiores detalhes, veja [1], por exemplo). Portanto a notação

$$
M^{(\infty)}(p) \stackrel{\text { def }}{=} \alpha(p)
$$

torna-se natural e com ela estabelecemos a seguinte

Definição 2.6 A probabilidade crítica $p_{c}^{(i)}$ para a familia de PCFTDs ${ }_{p} X_{\bullet}^{(i)}, i \in \overline{\mathbb{N}}$, é a única raiz real de $M^{(i)}(p)$ no intervalo $(0,1]$. Portanto

$$
M^{(i)}\left(p_{c}^{(i)}\right)=0, \forall i \in \overline{\mathbb{N}}
$$

A interpretação heurística da definição 2.6 é a seguinte:

(i) quando $p<p_{c}^{(i)}, \lim _{n \rightarrow \infty} \bar{X}_{n}^{(i)}=-\infty \quad q s$, ie a infecção desaparece com probabilidade um;

(ii) quando $p>p_{c}^{(i)}, \lim _{n \rightarrow \infty} \bar{p}_{n}^{(i)}=+\infty \quad q s$, ie a infecção se espalha por todo "eixo" $\mathbb{Z}$.

$\mathrm{Na}$ próxima seç̧ão provaremos um importante resultado que relaciona as probabilidades críticas $p_{c}^{(i)} \mathrm{s}$, $i \in \overline{\mathbb{N}}$, que acabamos de definir, qual seja " $p_{c}^{(i)} \nearrow_{i} p_{c}^{(\infty)} " 6$.

Esta convergência em forma não decrescente para a probabilidade crítica do processo de percolação orientada, o Teorema de Convergência, juntamente com a possibilidade de calcular as $p_{c}^{(i)} \mathrm{s}(i \in \mathbb{N})$ por meios algébricos ( Secção 4), é o ponto central deste trabalho.

\footnotetext{
${ }^{5} \alpha(p)=-\infty$, quando $p<p_{c} ;$ de modo que o comportamento estritamente crescente de $M^{(i)}(p)$ não se aplica precisamente a $\alpha(p)$.

${ }^{6} p_{c}^{(\infty)} \stackrel{\text { def }}{=} p_{c}$, conciliando as notaçōes das seç̧ões 1 e 2.
} 


\section{O Teorema de Convergência e Resultados Preliminares}

Lema $3.1 \frac{p \bar{X}_{n}^{(i)}}{n} \stackrel{(n)}{\longrightarrow} M^{(i)}(p) \quad$ q s $, \forall i \in \overline{\mathbb{N}}, p \in(0,1]$

Prova:

Primeiro caso $(i \in \mathbb{N})$ : Seja $n_{l}^{(i)}$ o número (aleatório) de visitas que a CMI ${ }_{p} Y_{\bullet}^{(i)}$ faz ao estado $\sigma_{l}$ até o instante $n$ (de tal sorte que $\sum_{l=0}^{2^{i}-1} n_{l}^{(i)}=n$ ) e $J_{l, k}^{(i)}$ a magnitude do késimo salto do PMD ${ }_{p} \bar{X}_{\bullet}^{(i)}$ na configuração $\sigma_{l}$.

A propriedade markoviana do PCFTD ${ }_{p} X_{\bullet}^{(i)}$ faz com que os $J_{l, k}^{(i)},: k \in\{1,2,3, \ldots\}$ sejam v.a.s iid com $\mathbb{E}\left[J_{l, k}^{(i)}\right]=M_{l}^{(i)}$.

Agora,

$$
\begin{gathered}
\frac{p}{\bar{X}_{n}^{(i)}}=\sum_{l=0}^{2^{i}-1} \sum_{k=1}^{n_{l}^{(i)}} \frac{J_{l, k}^{(i)}}{n}=\sum_{l=0}^{2^{i}-1} \sum_{k=1}^{n_{l}^{(i)}} \frac{J_{l, k}^{(i)}}{n_{l}^{(i)}} \frac{n_{l}^{(i)}}{n}=\sum_{l=0}^{2^{i}-1} \frac{n_{l}^{(i)}}{n} \sum_{k=1}^{n_{l}^{(i)}} \frac{J_{l, k}^{(i)}}{n_{l}^{(i)}} \\
\frac{n_{l}^{(i)}}{n} \stackrel{(n)}{\longrightarrow} \pi_{l}^{(i)} q s \quad[4, \text { pag.67] e, pela }
\end{gathered}
$$

Lei Forte de Kolmogorov: $\quad \sum_{k=1}^{n_{l}^{(i)}} \frac{J_{l, k}^{(i)}}{n_{l}^{(i)}} \stackrel{n_{l}^{(i)}}{\longrightarrow} M_{l}^{(i)}(p) q s \quad$ [5, pag.212]

Tomando-se o limite, para $n \rightarrow \infty$, e considerando a definição 2.5 , obtemos o resultado desejado.

Segundo caso $(i=\infty)$ : Veja [1, pag.1004].

Lema 3.2 Para $i \in \mathbb{N}$, cada função $M^{(i)}:(0,1] \rightarrow(-\infty, 1]$ é estritamente crescente (em $\left.p\right)$. Ademais,

$$
p \mapsto M^{(i)}(p)
$$

$M^{(i)}$ é uma sobrejeção de $(0,1]$ em $(-\infty, 1]$.

Prova:

O comportamento não decrescente de $M^{(i)}(p)$ segue do Acoplamento de Segunda Ordem (desigualdade 7 acima), bem como do lema 3.1, que acabamos de provar. As definições 2.2, 2.3, 2.4 e 2.5 fazem com que $M^{(i)}(p)$ seja uma função racional de $p$, tal que $M^{(i)}\left(0_{+}\right)=-\infty$ e $M^{(i)}(1)=(1)$; portanto $M^{(i)}(p)$ não pode ser constante em nenhum intervalo $\left[p_{1}, p_{2}\right] \subset(0,1]$, o que acarreta o comportamento estritamente crescente de $M^{(i)}(p)$.

Comentário sobre o lema 3.2 :

Neste ponto, é conveniente ressaltar que a função $\alpha(p) \stackrel{\text { def }}{=} M^{(\infty)}(p)$ é não decrescente e estritamente positiva, quando $p>p^{c}$; igual a zero, quando $p=p_{c}$ e infinitamente negativa, quando $p<p_{c}$. Novamente, a referência é [1]. O lema 3.2 conduz ao

Corolário 3.3 Para cada $i \in \overline{\mathbb{N}}, M^{(i)}$ tem apenas uma raiz real no intervalo $(0,1]$, que denotamos por $p_{c}^{(i)}$, de acordo com a Seç̧ão 2.4. 
Lema 3.4 A seqüência de funções $\left\{M^{(i)}(p)\right\}_{i \in \overline{\mathbb{N}}}$, é não crescente, ie $i \leq j ; i, j \in \mathbb{N} \Rightarrow M^{(i)}(p) \geq M^{(j)}(p), \forall p \in(0,1]$. Em particular $\alpha(p) \leq M^{(i)}(p) ; \forall i \in \mathbb{N}, \forall p \in(0,1]$. Prova:

O comportamento não crescente (em $i$ ) de $\left\{M^{(i)}(p)\right\}_{i \in \overline{\mathbb{N}}}$ segue do Acoplamento de Primeira Ordem (desigualdade 6 acima) e novamente do lema 3.1.

O lema 3.4 conduz ao

Corolário 3.5 A seqüência numérica $\left\{p_{c}^{(i)}\right\}_{i \in \mathbb{N}}$ é não decrescente. Desta forma $\lim _{i \rightarrow \infty} p_{c}^{(i)}$ está bem definido e $p_{c}^{(i)} \nearrow_{i} \lim _{i \rightarrow \infty} p_{c}^{(i)}$. Ademais $p_{c} \stackrel{\text { def }}{=} p_{c}^{(\infty)} \geq p_{c}^{(i)}, \forall i \in \mathbb{N}$.

e ao

Corolário $3.6 p_{c}^{(\infty)} \geq \lim _{i \rightarrow \infty} p_{c}^{(i)}$.

Agora, voltamos a atenção para a desigualdade reversa, qual seja " $p_{c} \leq \lim _{i \rightarrow \infty} p_{c}^{(i)}$ ". Para tal, considerem-se os seguintes espaços de probabilidade:

- $(\Omega, \mathbb{P})$ : o espaço de probabilidade abstrato, onde as v.a.s iid $\xi_{n m}^{j}$ foram definidas;

- $\left(\mathcal{S}^{(i)}, \pi^{(i)}\right)$ : o espaço de probabilidade finito das CMIs $Y_{\bullet}^{(i)}$, dotado de sua medida estacionária $\pi^{(i)}$

- $\left(\Omega \times \mathcal{S}^{(i)}, \mathbb{P} \times \pi^{(i)}\right)$ : o espaço produto.

No espaço produto, definimos os seguintes processos estocásticos:

- $\left\{{ }_{p} \overline{\mathcal{X}}_{n}^{(i)}\right\}_{n \in \mathbb{N}}$, por ${ }_{p} \overline{\mathcal{X}}_{n}^{(i)}((\omega, \sigma)) \stackrel{\text { def }}{=} \bar{X}_{n}^{(i)}(\omega), \forall(\omega . \sigma) \in \Omega \times \mathcal{S}^{(i)}$

- $\left\{\begin{array}{l}\pi^{(i)} \\ \mathcal{X}_{n}^{(i)}\end{array}\right\}_{n \in \mathbb{N}}$, por ${ }_{p}^{(i)} \overline{\mathcal{X}}_{n}^{(i)}((\omega, \sigma)) \stackrel{\text { def }}{=}{ }_{p}^{\eta_{\sigma}} \bar{X}_{n}^{(i)}(\omega), \forall(\omega . \sigma) \in \Omega \times \mathcal{S}^{(i)}$,

onde $\eta_{\sigma} \in\{0,1\}^{\mathcal{V}_{0}}$ é tal que $\eta_{\sigma}(2 m) \stackrel{\text { def }}{=}\left\{\begin{array}{cc}0 & , \quad \begin{array}{c}m>0 \\ \sigma(-2 m)\end{array} \\ 1 & , \quad-1 \leq m \leq-i \\ \end{array} ;\right.$ de tal sorte que a CMI ${ }_{p}^{\eta_{\sigma}} Y_{\bullet}^{(i)}$ começa na configuração $\sigma \in \mathcal{S}^{(i)}$.

Agora, estabelecemos o

Lema $3.7 \mathbb{E}\left[\frac{{ }_{p} \bar{X}_{n}^{(i)}}{n}\right] \geq M^{(i)}(p) ; \forall n, i \in \mathbb{N}, p \in(0,1]$.

Prova:

O Acoplamento de Terceira Ordem (desigualdade 9 acima) implica que

$$
\forall(\omega, \sigma) \in \Omega \times \mathcal{S}^{(i)},{ }_{p} \overline{\mathcal{X}}_{n}^{(i)}((\omega, \sigma))={ }_{p} \bar{X}_{n}^{(i)}(\omega) \geq{ }_{p}^{\eta_{\sigma}} \bar{X}_{n}^{(i)}(\omega)={ }_{p}^{\pi^{(i)}} \overline{\mathcal{X}}_{n}^{(i)}((\omega, \sigma)) .
$$

Integrando os dois lados da desigualdade acima (com respeito a $\mathbb{P} \times \pi^{(i)}$ ), obtemos:

$$
\mathbb{E}\left[{ }_{p} \overline{\mathcal{X}}_{n}^{(i)}\right]=\left(\mathbb{E}_{\mathbb{P}}\left[{ }_{p} \bar{X}_{n}^{(i)}\right]\right) \geq \mathbb{E}\left[\begin{array}{l}
\pi_{p}^{(i)} \\
\bar{X}_{n}^{(i)}
\end{array}\right]\left(=n M^{(i)}(p)\right),
$$

onde a última igualdade acima segue do fato de o processo $\left\{\begin{array}{l}\pi_{p}^{(i)} \\ \mathcal{X}_{n}^{(i)}\end{array}\right\}_{n \in \mathbb{N}}$ possuir incrementos estacionários de média $M^{(i)}(p)$. 


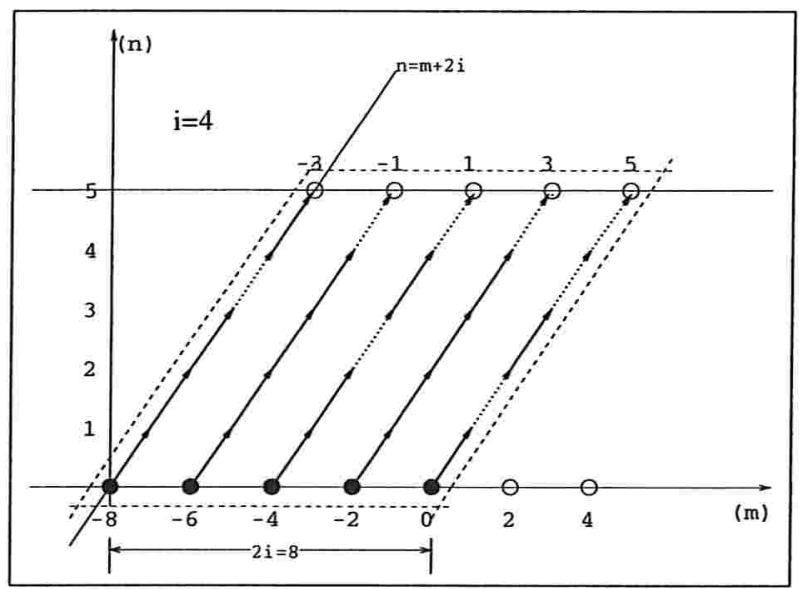

Figura 3: (Refere-se ao lema 3.8) Não pode haver infecção à força dentro do paralelograma tracejado. Os processos ${ }_{p} X_{\bullet}^{(4)}$ e ${ }_{p} X_{\bullet}^{(\infty)}$ são indistingüíveis nesta região.

Lema $3.8 \lim _{i \rightarrow \infty} \mathbb{E}\left[{ }_{p} \bar{X}_{n}^{(i)}\right]=\mathbb{E}\left[{ }_{p} \bar{X}_{n}^{(\infty)}\right]$ para todo $n \in \mathbb{N}($ fixo $)$ e $p \in(0,1]$.

Prova :

Como os saltos à direita são limitados por " +1 ", segue que $\forall \omega \in \Omega,{ }_{p} \bar{X}_{n}^{(i)} \leq n \Longrightarrow{ }_{p} \bar{X}_{n}^{(i)}-m \leq n-m$. Entretanto, para que o sítio $(n, m)$ seja infectado à força, devemos ter ${ }_{p} \bar{X}_{n}^{(i)}-m>2 i$ (Def. 2.1(b) acima). Portanto, se $n-m \leq 2 i$, então ${ }_{p} \bar{X}_{n}^{(i)}-m \leq 2 i, \forall \omega \in \Omega$ e o sítio $(n, m)$ não será infectado à força, para nenhuma realização $\omega \in \Omega$. Desta forma, podemos mostrar por indução sobre $n$ que “ $n-m \leq 2 i \Longrightarrow X_{n}^{(\infty)}(m)[\omega]=X_{n}^{(i)}(m)[\omega], \forall \omega \in \Omega$ ", ie todos os sítios $(n, m)$ tais que $n-m \leq 2 i$ têm o mesmo estado de infecção em relação aos processos ${ }_{p} X_{\bullet}^{(\infty)}$ e ${ }_{p} X_{\bullet}^{(i)}$. (Veja a figura 3 acima para o caso: $i=4)$.

Portanto, para cada instante $n$ (fixo),

$$
{ }_{p} \bar{X}_{n}^{(\infty)}<{ }_{p} \bar{X}_{n}^{(i)} \Longrightarrow{ }_{p} X_{n}^{(\infty)}(m)=0, \forall m \geq n-2 i
$$

O raciocínio que conduz a ( 13 ) é o seguinte:

$$
\begin{gathered}
{ }_{p} \bar{X}_{n}^{(i)} \geq n-2 i \Longrightarrow{ }_{p} X_{n}^{(\infty)}\left({ }_{p} \bar{X}_{n}^{(i)}\right)={ }_{p} X_{n}^{(i)}\left({ }_{p} \bar{X}_{n}^{(i)}\right) \stackrel{\text { def }}{=} 1 \stackrel{(6)}{\Longrightarrow} \\
{ }_{p} \bar{X}_{n}^{(i)} \stackrel{\text { def }}{=} \sup \left\{m \in \mathbb{Z}_{n}:{ }_{p} X_{n}^{(i)}(m)=1\right\}=\sup \left\{m \in \mathbb{Z}_{n}:{ }_{p} X_{n}^{(\infty)}(m)=1\right\} \stackrel{\text { def }}{=}{ }_{p} \bar{X}_{n}^{(\infty)} \Longrightarrow \\
\left\{{ }_{p} \bar{X}_{n}^{(i)} \geq n-2 i\right\} \subset\left\{{ }_{p} \bar{X}_{n}^{(i)}={ }_{p} \bar{X}_{n}^{(\infty)}\right\} \Longrightarrow\left\{{ }_{p} \bar{X}_{n}^{(i)} \neq{ }_{p} \bar{X}_{n}^{(\infty)}\right\} \subset\left\{{ }_{p} \bar{X}_{n}^{(i)}<n-2 i\right\} \subset\left\{{ }_{p} \bar{X}_{n}^{(\infty)}<n-2 i\right\} \\
\text { Assim, }(13) \text { segue ao identificarmos }\left\{{ }_{p} \bar{X}_{n}^{(i)} \neq{ }_{p} \bar{X}_{n}^{(\infty)}\right\} \operatorname{com}\left\{{ }_{p} \bar{X}_{n}^{(i)}>{ }_{p} \bar{X}_{n}^{(\infty)}\right\} \text { e }\left\{{ }_{p} \bar{X}_{n}^{(\infty)}<n-2 i\right\} \\
\operatorname{com}\left\{{ }_{p} X_{n}^{(\infty)}(m)=0, \forall m \geq n-2 i\right\} .
\end{gathered}
$$


Agora, observe que, se a infecção não está presente na região $\left\{(n, m) \in \mathcal{V}_{n}: m \geq n-2 i\right\}$ da fatia $\mathcal{V}_{n}$, todos os caminhos que ligam os seus sítios à fatia $\mathcal{V}_{0}$ devem estar interrompidos em algum lugar. Em particular, as $(i+1)$ retas que ligam o sítio $(0,-2 j)$ ao sítio $(n, n-2 j)$, para $0 \leq j \leq i$, devem estar obstruídas em algum ponto (Figura 3 acima). Como estas retas são constituídas de elos diferentes (portanto independentes, por construção ), a probabilidade deste evento será dada por $\left(1-p^{n}\right)^{i+1}$, e então teremos:

$$
\mathbb{P}\left({ }_{p} \bar{X}_{n}^{(i)} \neq{ }_{p} \bar{X}_{n}^{(\infty)}\right) \leq \mathbb{P}\left({ }_{p} X_{n}^{(\infty)}(m)=0, \forall m \geq n-2 i\right) \leq\left(1-p^{n}\right)^{i+1}
$$

De sorte que ${ }_{p} \bar{X}_{n}^{(i)} \stackrel{i}{\longrightarrow}{ }_{p} \bar{X}_{n}^{(\infty)}$ em probabilidade, e existirá uma sub-seqüência $\left(i_{k}\right)_{k \in \mathbb{N}}$ tal que ${ }_{p} \bar{X}_{n}^{\left(i_{k}\right)} \stackrel{k}{\longrightarrow} \bar{X}_{n}^{(\infty)} q s \quad\left[6\right.$, Teorema 7.6]. Como toda a seqüência $\left\{{ }_{p} \bar{X}_{n}^{(i)}\right\}_{i \in \mathbb{N}}$ é não-crescente (em $i$ ), devemos ter ${ }_{p} \bar{X}_{n}^{(i)} \stackrel{i}{\longrightarrow}{ }_{p} \bar{X}_{n}^{(\infty)} q s$ da mesma forma, e podemos invocar o Teorema da Convergência Monótona para concluir que $\mathbb{E}\left[{ }_{p} \bar{X}_{n}^{(i)}\right] \searrow^{i} \mathbb{E}\left[{ }_{p} \bar{X}_{n}^{(\infty)}\right], \forall n \in \mathbb{N}$.

Finalmente, estamos em condições de provar o Teorema de Convergência:

Teorema $3.9 p_{c}^{(i)} \nearrow_{i} p_{c}$

Prova : Suponha que $\lim _{i \rightarrow \infty} p_{c}^{(i)}<p_{c}$, de modo que se possa escolher $p$ tal que $\lim _{i \rightarrow \infty} p_{c}^{(i)}<p<p_{c}$. Neste caso o lema 3.2 e o corolário 3.5 nos asseguram que $\forall i \in \mathbb{N}, M^{(i)}(p)>M^{(i)}\left(p_{c}^{(i)}\right) \stackrel{\text { def }}{=} 0$ e o lema 3.7 que $\mathbb{E}\left[{ }_{p} \bar{X}_{n}^{(i)}\right] \geq n M^{(i)}(p)>0, \forall i \in \mathbb{N}, \forall n \in \mathbb{N}$. Desta forma

$$
\lim _{i \rightarrow \infty} \mathbb{E}\left[\bar{X}_{n}^{(i)}\right] \geq 0, \forall n \in \mathbb{N}
$$

, onde a desigualdade 6 nos assegura que o limite acima está bem definido.

Por outro lado,

$$
\begin{array}{rlr}
p<p_{c} & \Longrightarrow \frac{{ }_{p} \bar{X}_{n}^{(\infty)}}{n} \stackrel{(n)}{\longrightarrow}-\infty q s & \text { lema } 3.1 \text { e comentário sobre o lema } 3.2 \\
& \Longrightarrow \mathbb{E}\left[\frac{{ }_{p} \bar{X}_{n}^{\infty}}{n}\right] \stackrel{(n)}{\longrightarrow}-\infty & \text { Lema de Fatou } \\
& \Longrightarrow \exists \bar{n}: \mathbb{E}\left[{ }_{p} \bar{X}_{\bar{n}}^{\infty}\right]<0 &
\end{array}
$$

Aplicando o lema 3.8 para $\bar{n}$ (fixo), obtemos:

$$
\lim _{i \rightarrow \infty} \mathbb{E}\left[{ }_{p} \bar{X}_{\bar{n}}^{(i)}\right]=\mathbb{E}\left[{ }_{p} \bar{X}_{\bar{n}}^{\infty}\right]<0
$$

o que contradiz a desigualdade 14 acima. Portanto, $\lim _{i \rightarrow \infty} p_{c}^{(i)} \geq p_{c}$ e o teorema segue do corolário 3.6. 


\section{Determinação das Probabilidades Críticas}

\subsection{Determinação Algébrica das Probabilidades Críticas}

O teorema 3.9 ( $O$ Teorema de Convergência) possui interesse teórico per se. Sem embargo, resultados mais fracos como os corolários 3.5 e 3.6 já nos mostram que cada $p_{c}^{(i)}, i \in \mathbb{N}$, é uma quota inferior (refinada em relação às suas predecessoras) para a probabilidade crítica do Processo de Percolação Orientada.

Apesar de já estar implícito nas secções 2.3 .3 e 2.4 acima, apresentamos a seguir o algoritmo que conduz ao cálculo exato das probabilidades críticas $p_{c}^{(i)}, i \in \mathbb{N}$.

De acordo com as definições $2.2,2.3,2.4,2.5$ e 2.6 , as probabilidades críticas $p_{c}^{(i)}, i \in \mathbb{N}$, podem ser determinadas nos seguintes passos:

(i) Determinação das Probabilidades $P_{(l, m, k)}^{(i)}$ (Def. 2.3) em Função de $q \stackrel{\text { def }}{=} 1-p$ :

um cálculo combinatório elementar mostra que as probabilidades $P_{(l, m, k)}^{(i)}, 0 \leq l, m \leq 2^{i}-1, k \in \mathbb{N}$, podem ser expressas como funções polinomiais de $q$, ie

$$
P_{(l, m, k)}^{(i)}=P_{(l, m, k)}^{(i)}(q) \text { : polinômio em } q
$$

(ii) Determinação da Matriz de Transição $\left(q_{l m}^{(i)}\right)_{0 \leq l, m \leq 2^{i}-1}$ da CMI ${ }_{p} Y_{\bullet}^{(i)}$ :

as probabilidades de transição definidas em 11 podem ser expressas como

$$
q_{l m}^{(i)}=\sum_{k=0}^{\infty} P_{(l, m, k)}^{(i)}(q) .
$$

Como a seqüência numérica $\left(P_{(l, m, k)}^{(i)}(q)\right)_{k \in \mathbb{N}}$ forma uma progressão geométrica (de razão $q^{2}$ ) a partir do $(i+2)^{\text {ésimo }}$ termo, as probabilidades de transição $q_{l m}^{(i)}$ podem ser expressas como funções racionais de $q$. Em verdade, estas probabilidades serão sempre polinômios em $q$ :

$$
q_{l m}^{(i)}=q_{l m}^{(i)}(q): \text { polinômio em } q
$$

(iii) Determinação da Medida Estacionária $\left(\pi^{i}\right)$ da CMI ${ }_{p} Y_{\bullet}^{(i)}$ :

o cáculo combinatório que conduziu à equação 15 nos mostra que as probabilidades de transição $q_{l m}^{(i)}(q), 0 \leq l, m \leq 2^{i}-1$, são estritamente positivas, para $q \in(0,1)$, de sorte que a matriz de transição $\left(q_{l m}^{(i)}\right)$ é irredutível e aperiódica. Portanto $\pi^{i}$ é uma medida de probabilidade bem definida em $\mathcal{S}^{i}$ e pode ser determinada algebricamente a partir de $\left(q_{l m}^{(i)}\right)$. Como conseqüência temos que:

$$
\pi_{l}^{(i)}(q) \text { : função racional estritamente positiva de } q, q \in(0,1), 0 \leq l \leq 2^{i}-1
$$

(iv) Determinação de $M_{l}^{(i)}$, o Salto Médio do PCFTD ${ }_{p} X_{\bullet}^{(i)}$ na Configuração $\sigma_{l}$ : A definição 2.4 e o fato de a seqüência $\left(P_{l m k}^{(i)}(q)\right)_{k \in \mathbb{N}}$ ser essencialmente uma progressão geométrica acarretam que

$$
M_{l}^{(i)}(q) \text { : função racional de } q, 0 \leq l \leq 2^{i}-1
$$


(v) Determinação de $M^{(i)}$, o Salto Médio do PCFTD ${ }_{p} X_{\bullet}^{(i)}$ :

A definição 2.5 e os passos (iii) e (iv) acima asseguram que

$$
M^{(i)}(q) \text { : função racional de } q
$$

(vi) Determinação da Probabilidade Crítica $p_{c}^{(i)}$ :

De acordo com o corolário 3.3 e a definição $2.6, p_{c}^{(i)}$ é a única raiz real de $M^{(i)}(p)$ no intervalo $(0,1)$; $\mathrm{e}$, portanto, a única raiz real do polinômio numerador de $M^{(i)}(p)$. Desta forma, a sua determinação se obtém numericamente.

No que segue, empregaremos o algoritmo descrito acima para calcular algebricamente as primeiras probabilidades críticas:

\subsubsection{A Probabilidade Crítica de Ordem Zero, $p_{c}^{(0)}$ :}

Neste caso $\left|\mathcal{S}^{(i)}\right|=1$, de modo que a CMI ${ }_{p} Y_{\bullet}^{(i)}$ é trivial:

$$
\begin{aligned}
M^{(0)}(q) & =M_{0}^{(0)}(q)=\sum_{k=0}^{\infty}(1-2 k) \cdot P_{0,0, k}^{(0)}(q)=1 \cdot p-1 \cdot q\left(1-q^{2}\right)-3 \cdot q^{3}\left(1-q^{2}\right)-5 \cdot q^{5}\left(1-q^{2}\right)-\ldots \\
& =(1-q)-q\left(1-q^{2}\right) \cdot\left[1+3 q^{2}+5 q^{4}+7 q^{6}+\ldots\right]=(1-q)-q\left(1-q^{2}\right) \frac{1+q^{2}}{\left(1-q^{2}\right)^{2}}= \\
& =(1-q)-\frac{q+q^{3}}{1-q^{2}}=\frac{1-2 q-q^{2}}{1-q^{2}}
\end{aligned}
$$

Portanto,

$$
M^{(0)}(q)=0 \Leftrightarrow 1-2 q-q^{2}=0 \Rightarrow p_{c}^{(0)}=2-\sqrt{2}=0.58579 \ldots
$$

4.1.2 A Probabilidade Crítica de Primeira Ordem, $p_{c}^{(1)}$ :

$$
\begin{gathered}
\left(q_{l m}^{(1)}\right)(q)=\left[\begin{array}{cc}
q-q^{3}+q^{4} & 1-q+q^{3}-q^{4} \\
q^{2} & 1-q^{2}
\end{array}\right], \quad \pi_{l}^{(1)}(q)=\frac{\left(q^{2}, 1-q+q^{3}-q^{4}\right)}{1-q+q^{2}+q^{3}-q^{4}} \\
M_{0}^{(1)}(q)=\frac{1-2 q-3 q^{2}+2 q^{4}}{1-q^{2}}, \quad M_{1}^{(1)}(q)=\frac{1-2 q-q^{2}}{1-q^{2}} \\
M^{(1)}(q)=\frac{1-3 q+2 q^{2}-6 q^{4}+q^{5}+3 q^{6}}{1-q+2 q^{3}-2 q^{4}-q^{5}+q^{6}}
\end{gathered}
$$

Portanto,

$$
M^{(1)}(q)=0 \Rightarrow p_{c}^{(1)}=0.604233 \ldots
$$




\subsubsection{A Probabilidade Crítica de Segunda Ordem, $p_{c}^{(2)}$ :}

$$
\begin{aligned}
& \left(q_{l m}^{(2)}(q)\right)=\left[\begin{array}{cccc}
q-q^{3}+q^{6} & q-2 q^{2}+q^{3}+q^{4}-q^{6} & 1-2 q+q^{2}+q^{4}-q^{6} & q^{2}-2 q^{4}+q^{6} \\
q^{2}-q^{3}+q^{4}-q^{5}+q^{6} & q-q^{2}+q^{5}-q^{6} & q-2 q^{2}+2 q^{3}-q^{4}+q^{5}-q^{6} & 1-2 q+2 q^{2}-q^{3}-q^{5}+q^{6} \\
2 q^{3}-q^{4}-q^{5}+q^{6}-q^{7}+q^{8} & 2 q^{2}-3 q^{3}+q^{4}+q^{7}-q^{8} & q-2 q^{3}+2 q^{5}-q^{6}+q^{7}-q^{8} & 1-q-2 q^{2}+3 q^{3}-q^{5}-q^{7}-q^{8} \\
q^{4} & q^{2}-q^{4} & q^{2}-q^{4} & 1-2 q^{2}+q^{4}
\end{array}\right] \\
& \pi_{\mathrm{o}}^{(2)}(q)=\frac{-2 q^{4}+2 q^{5}-4 q^{6}+9 q^{7}-14 q^{8}+15 q^{9}-9 q^{10}+2 q^{11}}{-1+3 q-6 q^{2}+8 q^{3}-17 q^{4}+30 q^{5}-44 q^{6}+46 q^{7}-20 q^{8}-17 q^{9}+38 q^{10}-32 q^{11}+13 q^{12}-2 q^{13}} \\
& \pi_{1}^{(2)}(q)=\frac{-q^{2}+2 q^{3}-2 q^{4}+q^{5}-4 q^{6}+9 q^{7}-5 q^{8}-6 q^{9}+12 q^{10}-8 q^{11}+2 q^{12}}{-1+3 q-6 q^{2}+8 q^{3}-17 q^{4}+30 q^{5}-44 q^{6}+46 q^{7}-20 q^{8}-17 q^{9}+38 q^{10}-32 q^{11}+13 q^{12}-2 q^{13}} \\
& \pi_{2}^{(2)}(q)=\frac{-q^{2}+q^{3}+2 q^{5}-9 q^{6}+15 q^{7}-14 q^{8}+8 q^{9}-2 q^{10}}{-1+3 q-6 q^{2}+8 q^{3}-17 q^{4}+30 q^{5}-44 q^{6}+46 q^{7}-20 q^{8}-17 q^{9}+38 q^{10}-32 q^{11}+13 q^{12}-2 q^{13}} \\
& \pi_{3}^{(2)}(q)=\frac{-1+3 q-4 q^{2}+5 q^{3}-13 q^{4}+25 q^{5}-27 q^{6}+13 q^{7}+13 q^{8}-34 q^{9}+37 q^{10}-26 q^{11}+11 q^{12}-2 q^{13}}{-1+3 q-6 q^{2}+8 q^{3}-17 q^{4}+30 q^{5}-44 q^{6}+46 q^{7}-20 q^{8}-17 q^{9}+38 q^{10}-32 q^{11}+13 q^{12}-2 q^{13}} \\
& M_{l}^{(2)}=\frac{1}{1-q^{2}}\left(1-2 q-5 q^{2}+4 q^{4}, 1-2 q-3 q^{2}+2 q^{4}, 1-2 q-q^{2}-2 q^{4}+2 q^{6}, 1-2 q-q^{2}\right) \\
& M^{(2)}(q)=\frac{-1+5 q-11 q^{2}+17 q^{3}-25 q^{4}+52 q^{5}-75 q^{6}+96 q^{7}-58 q^{8}-69 q^{9}+152 q^{10}-111 q^{11}-5 q^{12}+74 q^{13}-49 q^{14}+10 q^{15}}{\left(1-q^{2}\right)\left(-1+3 q-6 q^{2}+8 q^{3}-17 q^{4}+30 q^{5}-44 q^{6}+46 q^{7}-20 q^{8}-17 q^{9}+38 q^{10}-32 q^{11}+13^{12}-2 q^{13}\right)} .
\end{aligned}
$$

Portanto,

$$
M^{(2)}(q)=0 \Rightarrow p_{c}^{(2)}=0.614187
$$

\subsection{Determinação Numérica das Probabilidades Críticas:}

Os cálculos combinatórios que levaram à matriz de transição $\left(q^{(i)}\right)_{l m}$ e ao vetor de " mean-drifts" $M(i)_{l}$, descritos na seç̧ão 4.1 ; apesar de longos e fastidiosos para seres humanos, são prontamente executáveis por um computador digital: enquanto levamos toda uma tarde para calcular a matriz de transição $\left(q^{(2)}\right)_{l m}$ algebricamente (16 entradas polinomiais), o programa em FORTRAN 77 apresentado no Apêndice A, executado a uma freqüência de $600 \mathrm{MHz}$, calculou a matriz de transição $\left(q^{(9)}\right)_{l m}$ ( $4^{9}$ entradas polinomiais) em menos de um minuto!

O maior problema na codificação do algoritmo descrito na secção 4.1 numa linguagem computacional encontra-se precisamente no passo (iii), ou seja na determinação algébrica da medida estacionária $\pi^{(i)}$ , a partir da matriz de transição $\left(q^{(i)}\right)_{l m}(q)$. Embora isto possa ser feito diretamente, tratando os polinômios como se fossem entradas numéricas; se não levarmos em consideração as possíveis e prováveis simplificações algébricas que possam ocorrer, o grau dos polinômios resultantes logo se torna computacionalmente intratável. Infelizmente, encontrar estas simplificações está longe de ser um problema trivial.

Desta forma, adotamos uma abordagem diferente:

(i) Uma vez obtida algebricamente a matriz de transição $\left(q^{(i)}\right)_{l m}(q)$ e o vetor de " mean-drifts" $M_{l}^{(i)}(q)$, o programa gerou uma matriz de transição numérica e um vetor de "mean-drifts" numérico para uma seqüência decrescente de valores de $q$.

(ii) A partir de cada matriz de transição numérica, obteve-se numericamente a correspondente medida estacionária, resolvendo-se um sistema de equações lineares. Nesta etapa, a técnica de condensação pivotal ( "partial pivoting ", como descrita em [7] ) foi de fundamental importância no controle dos erros numéricos. 
(iii) O valor numérico de $M^{(i)}(q)$ foi então obtido, executando-se o produto escalar da definição 2.5 .

(iv) De acordo com a definição $2.6 q_{c}^{(i)}\left(\stackrel{\text { def }}{=} 1-p_{c}^{(i)}\right)$ estará entre o último valor de $q$ para o qual $M^{(i)}(q)$ foi negativo e o primeiro valor de $q$ para o qual $M^{(i)}(q)$ foi positivo.

Esta abordagem tem o ônus de introduzir erros numéricos (basicamente na etapa ii acima), dos quais a abordagem puramente algébrica da secção 4.1 esteve isenta. Portanto, para obtermos resultados numéricos confiáveis para as probabilidades críticas, limites superiores rigorosos para os erros numéricos devem ser fornecidos. Para tal, seguimos o procedimento conhecido como "foward analysis" descrito em [7], o qual consiste basicamente em obter quotas superiores parciais para os erros numéricos envolvidos em cada operação aritmética executada na seqüência lógica de cálculos que conduziu ao valor numérico de $M^{(i)}(q)$. Este procedimento, apesar de aparentemente primitivo, tem a vantagem de fornecer uma limitação segura e rigorosa para o erro numérico envolvido a partir da etapa (ii) acima.

O programa computacional que implementou em linguagem FORTRAN 77 os passos descritos acima, bem como o procedimento " forward analysis" para controle de erros numéricos, encontra-se listado no Apêndice A. Apresentamos abaixo um resumo dos resultados numéricos por ele gerados ${ }^{7}$ :

\begin{tabular}{|c|c|c|}
\multicolumn{3}{c}{$\mathrm{i}=1$} \\
\hline $\mathrm{p}$ & $M_{\text {Num }}^{(1)}(p)$ & $\Delta M^{(1)}(p)$ \\
\hline 0.604231 & $-9.15 \mathrm{E}-6$ & $6.2 \mathrm{E}-16$ \\
0.604232 & $-5.27 \mathrm{E}-6$ & $6.2 \mathrm{E}-16$ \\
0.604233 & $-1.40 \mathrm{E}-6$ & $6.2 \mathrm{E}-16$ \\
0.604234 & $+2.47 \mathrm{E}-6$ & $6.2 \mathrm{E}-16$ \\
\hline
\end{tabular}

\begin{tabular}{|c|c|c|}
\hline \multicolumn{3}{c}{$\mathrm{i}=2$} \\
$\mathrm{p}$ & $M_{N u m}^{(2)}(p)$ & $\Delta M^{(2)}(p)$ \\
\hline 0.614185 & $-1.01 \mathrm{E}-5$ & $6.1 \mathrm{E}-15$ \\
0.614186 & $-5.85 \mathrm{E}-6$ & $6.2 \mathrm{E}-15$ \\
0.614187 & $-1.57 \mathrm{E}-6$ & $6.1 \mathrm{E}-15$ \\
0.614188 & $+2.71 \mathrm{E}-6$ & $6.2 \mathrm{E}-15$ \\
\hline
\end{tabular}

\begin{tabular}{|c|c|c|}
\multicolumn{3}{c}{$\mathrm{i}=3$} \\
\hline $\mathrm{p}$ & $M_{N u m}^{(3)}(p)$ & $\Delta M^{(3)}(p)$ \\
\hline 0.620203 & $-1.36 \mathrm{E}-5$ & $2.5 \mathrm{E}-14$ \\
0.620204 & $-8.96 \mathrm{E}-6$ & $2.5 \mathrm{E}-14$ \\
0.620205 & $-4.32 \mathrm{E}-6$ & $2.6 \mathrm{E}-14$ \\
0.620206 & $+3.15 \mathrm{E}-7$ & $2.6 \mathrm{E}-14$ \\
\hline
\end{tabular}

\begin{tabular}{|c|c|c|}
\hline \multicolumn{3}{|c}{$\mathrm{i}=4$} \\
$\mathrm{p}$ & $M_{\text {Num }}^{(4)}(p)$ & $\Delta M^{(4)}(p)$ \\
\hline 0.624210 & $-9.52 \mathrm{E}-6$ & $8.0 \mathrm{E}-14$ \\
0.624211 & $-4.57 \mathrm{E}-6$ & $8.0 \mathrm{E}-14$ \\
0.624212 & $+3.71 \mathrm{E}-7$ & $8.0 \mathrm{E}-14$ \\
& & \\
\hline
\end{tabular}

\begin{tabular}{|c|c|c|}
\hline \multicolumn{3}{c}{$\mathrm{i}=5$} \\
$\mathrm{p}$ & $M_{N u m}^{(5)}(p)$ & $\Delta M^{(5)}(p)$ \\
\hline 0.627064 & $-1.31 \mathrm{E}-5$ & $1.9 \mathrm{E}-13$ \\
0.627065 & $-7.85 \mathrm{E}-6$ & $1.9 \mathrm{E}-13$ \\
0.627066 & $-2.63 \mathrm{E}-6$ & $1.9 \mathrm{E}-13$ \\
0.627067 & $+2.60 \mathrm{E}-6$ & $1.9 \mathrm{E}-13$ \\
\hline
\end{tabular}

\begin{tabular}{|c|c|c|}
\multicolumn{3}{c}{$\mathrm{i}=6$} \\
\hline $\mathrm{p}$ & $M_{\text {Num }}^{(6)}(p)$ & $\Delta M^{(6)}(p)$ \\
\hline 0.629201 & $-1.55 \mathrm{E}-5$ & $3.8 \mathrm{E}-13$ \\
0.629202 & $-9.98 \mathrm{E}-6$ & $3.8 \mathrm{E}-13$ \\
0.629203 & $-4.51 \mathrm{E}-6$ & $3.9 \mathrm{E}-13$ \\
0.629204 & $+9.66 \mathrm{E}-7$ & $3.8 \mathrm{E}-13$ \\
\hline
\end{tabular}

\begin{tabular}{|c|c|c|}
\multicolumn{3}{c}{$\mathrm{i}=7$} \\
\hline $\mathrm{p}$ & $M_{N u m}^{(7)}(p)$ & $\Delta M^{(7)}(p)$ \\
\hline 0.630863 & $-1.01 \mathrm{E}-5$ & $7.2 \mathrm{E}-13$ \\
0.630864 & $-4.41 \mathrm{E}-6$ & $7.2 \mathrm{E}-13$ \\
0.630865 & $+1.30 \mathrm{E}-6$ & $7.2 \mathrm{E}-13$ \\
& & \\
\hline
\end{tabular}

\begin{tabular}{|c|c|c|}
\hline \multicolumn{3}{|c}{$\mathrm{i}=8$} \\
$\mathrm{p}$ & $M_{N u m}^{(8)}(p)$ & $\Delta M^{(8)}(p)$ \\
\hline 0.632192 & $-8.53 \mathrm{E}-6$ & $1.3 \mathrm{E}-12$ \\
0.632193 & $-2.60 \mathrm{E}-6$ & $1.2 \mathrm{E}-12$ \\
0.632194 & $+3.32 \mathrm{E}-6$ & $1.3 \mathrm{E}-12$ \\
& & \\
\hline
\end{tabular}

\begin{tabular}{|c|c|c|}
\hline \multicolumn{3}{c}{$\mathrm{i}=9$} \\
\hline $\mathrm{p}$ & $M_{N u m}^{(9)}(p)$ & $\Delta M^{(9)}(p)$ \\
\hline 0.63326 & $-1.29 \mathrm{E}-4$ & $2.0 \mathrm{E}-12$ \\
0.63327 & $-6.79 \mathrm{E}-5$ & $2.0 \mathrm{E}-12$ \\
0.63328 & $-6.68 \mathrm{E}-6$ & $2.0 \mathrm{E}-12$ \\
0.63329 & $+5.45 \mathrm{E}-5$ & $2.0 \mathrm{E}-12$ \\
\hline
\end{tabular}

\footnotetext{
${ }^{7}$ os cálculos numéricos foram executados em dupla precisão .

${ }^{8}\left|M^{(i)}(p)-M_{N u m}^{(i)}(p)\right| \leq \Delta M^{(i)}(p) ;$ onde $M_{N u m}^{(i)}(p)$ denota o valor numérico obtido para o mean-drift de $i$ ésima ordem.
} 
A partir dos dados listados acima, as dez primeiras probabilidades críticas podem ser obtidas:

\begin{tabular}{||c|c||}
\hline \hline$i$ & $p_{c}^{(i)}$ \\
\hline 0 & 0,585787 \\
1 & 0,604233 \\
2 & 0,614187 \\
3 & 0,620205 \\
4 & 0,624211 \\
5 & 0,627066 \\
6 & 0,629203 \\
7 & 0,630864 \\
8 & 0,632193 \\
9 & 0,63328 \\
\hline \hline
\end{tabular}

Tabela 1: Probabilidades críticas obtidas numericamente.

Desta forma, estamos em condições de estabelecer a desigualdade anunciada na Introdução, qual seja:

$$
p_{c} \geq 0.63328
$$




\section{Simulações}

\subsection{Obtenção de Quotas Inferiores via Simulação}

O programa em FORTRAN 77 apresentado no Apêndice B à página 46 realiza uma trajetória $\omega$ do PCTDF ${ }_{p} X_{\bullet}^{(i)}$. A cada passo o valor de ${ }_{p} \bar{X}_{n}^{(i)} / n$ é calculado. Como prevê o Lema 3.1, este valor tende assintoticamente para $M^{(i)}(p)$ à medida que $n$ cresce. Desta forma, executando-se o programa para diferentes valores de $p$, pode-se estimar o valor de $p_{c}^{(i)}$ observando-se a altura do platô que se estabelece com o aumento de $n$ :

(a) altura do platô $<0 \Rightarrow p<p_{c}^{(i)}$,

(b) altura do platô $>0 \Rightarrow p>p_{c}^{(i)}$.

A tabela abaixo sintetiza os resultados obtidos nas simulações que vem a seguir: ${ }^{9}$

\begin{tabular}{||c|c||}
\hline \hline$i$ & $p_{c}^{(i)}$ \\
\hline 5 & 0,627 \\
6 & 0,629 \\
9 & 0,6332 \\
20 & 0,638 \\
40 & 0,641 \\
100 & 0,643 \\
200 & 0,6438 \\
1000 & 0,6445 \\
\hline \hline
\end{tabular}

Tabela 2: Probabilidades críticas obtidas por simulação .

É interessante comparar os dados da tabela 2 (obtidos por simulação) com os dados da tabela 1 (obtidos teoricamente).

\footnotetext{
${ }^{9}$ Veja o gráfico na figura 19 (pag. 33) para o caso $i=6$.
} 
5.1.1 Determinação de $p_{c}^{(5)}$

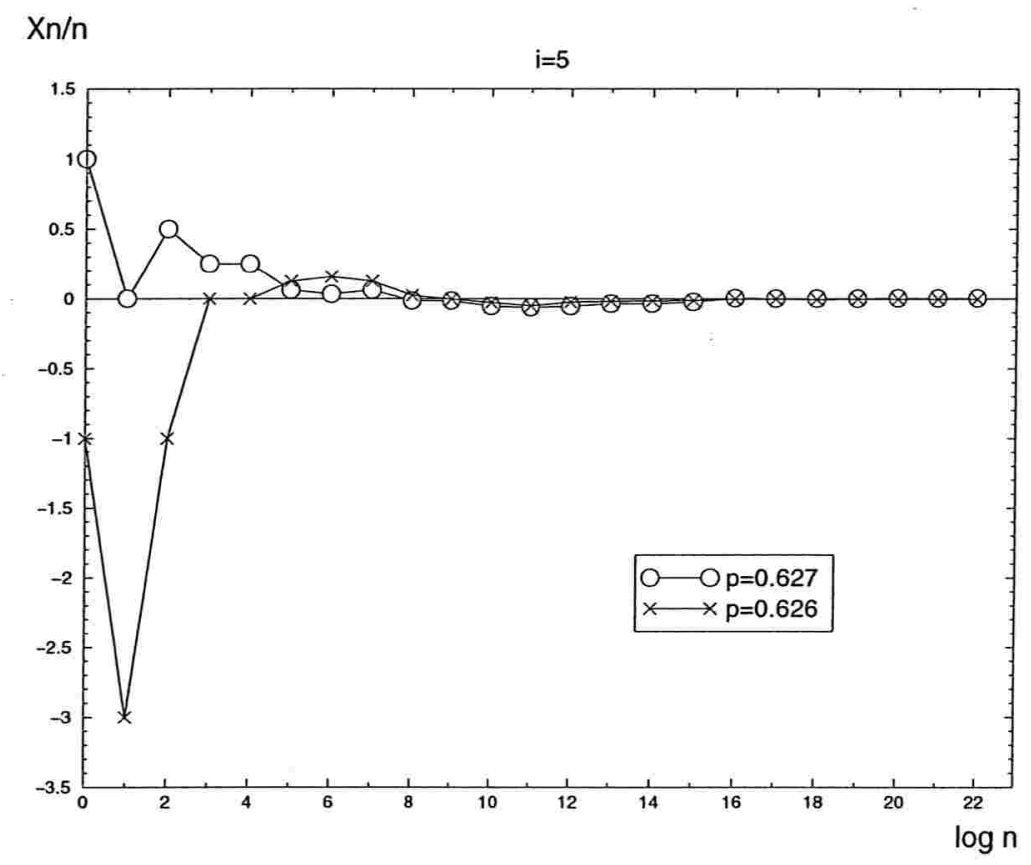

Figura 4: Realizações não acopladas dos processos ${ }_{.626} \bar{X}_{n}^{(5)} / n$ e ${ }_{.627} \bar{X}_{n}^{(5)} / n$.

Obs.: neste e nos demais gráficos, a escala horizontal é logarítmica de base 2 . 


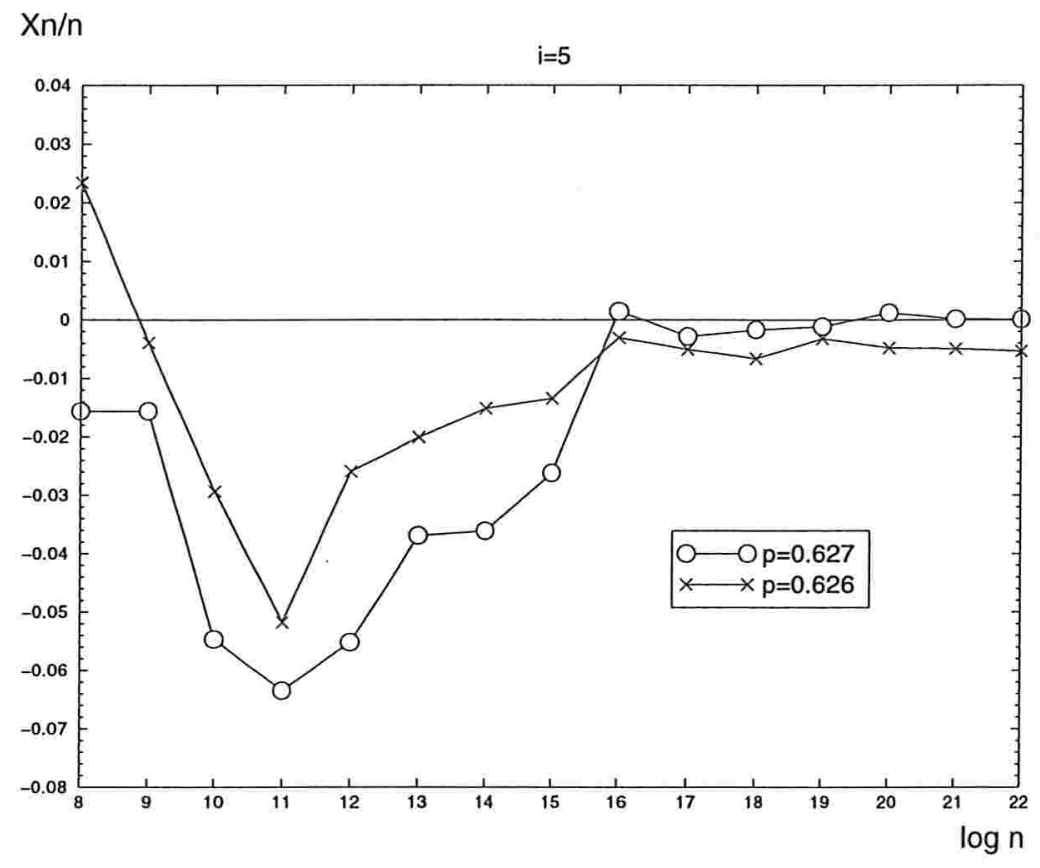

Figura 5: Ampliação e detalhe do gráfico na figura 4 . 
5.1.2 Determinação de $p_{c}^{(9)}$

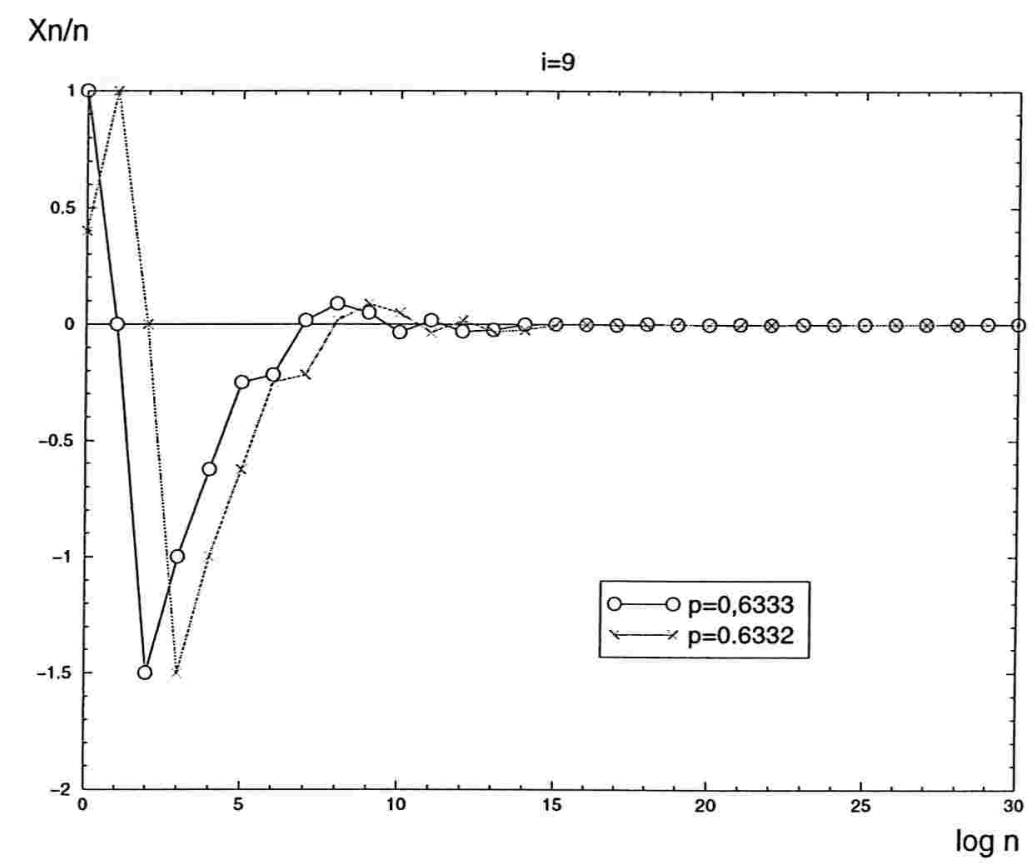

Figura 6: Realizações não acopladas dos processos ${ }_{.6332} \bar{X}_{n}^{(9)} / n$ e ${ }_{.6333} \bar{X}_{n}^{(9)} / n$. 


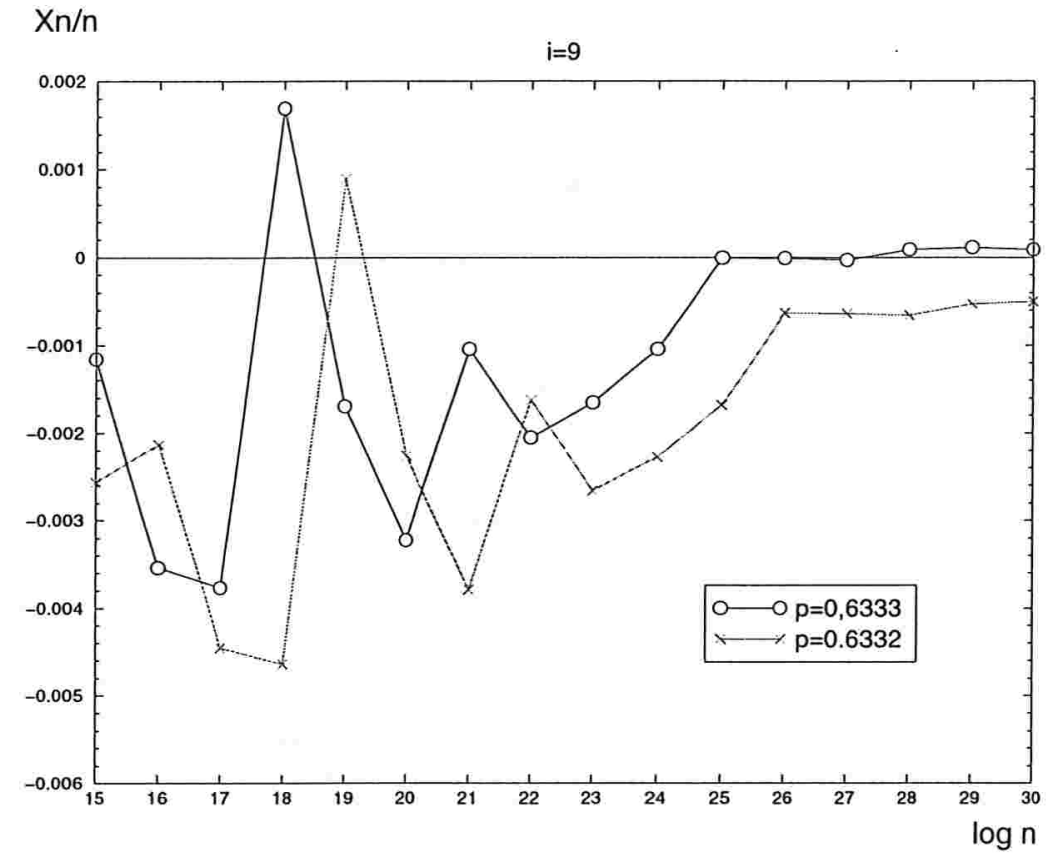

Figura 7: Ampliação e detalhe do gráfico na figura 6. 


\subsubsection{Determinação de $p_{c}^{(20)}$}

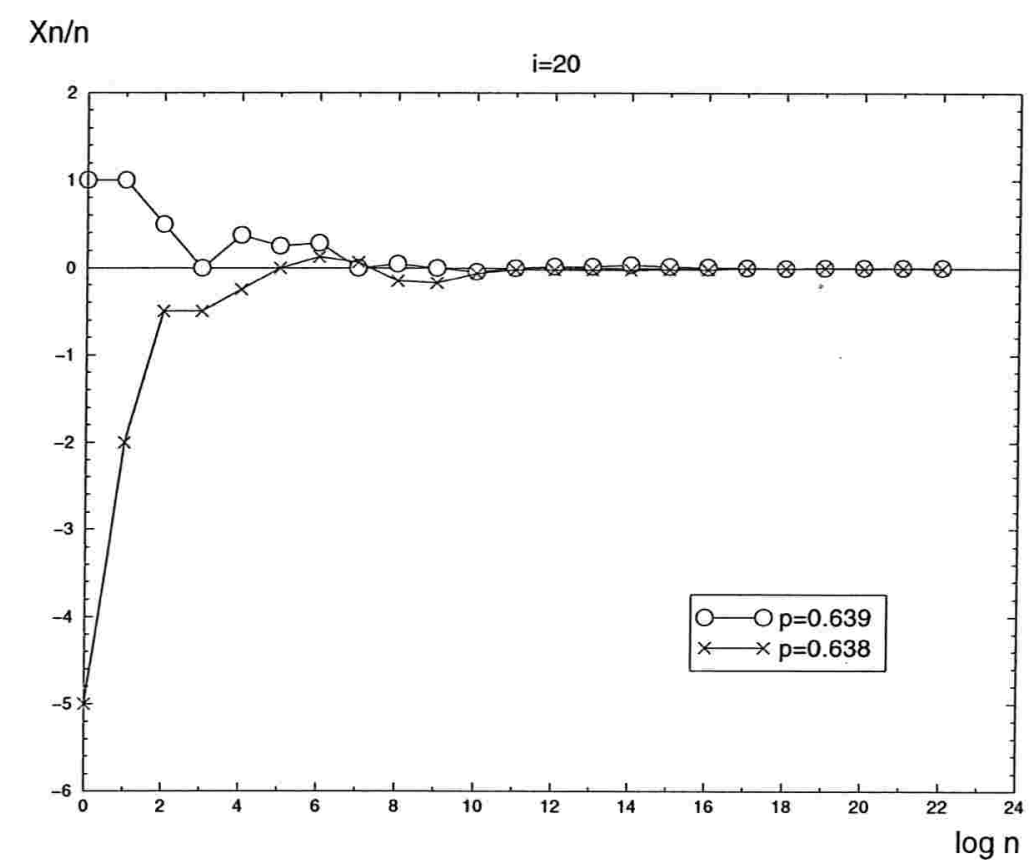

Figura 8: Realizações não acopladas dos processos ${ }_{.638} \bar{X}_{n}^{(20)} / n$ e ${ }_{.639} \bar{X}_{n}^{(20)} / n$. 


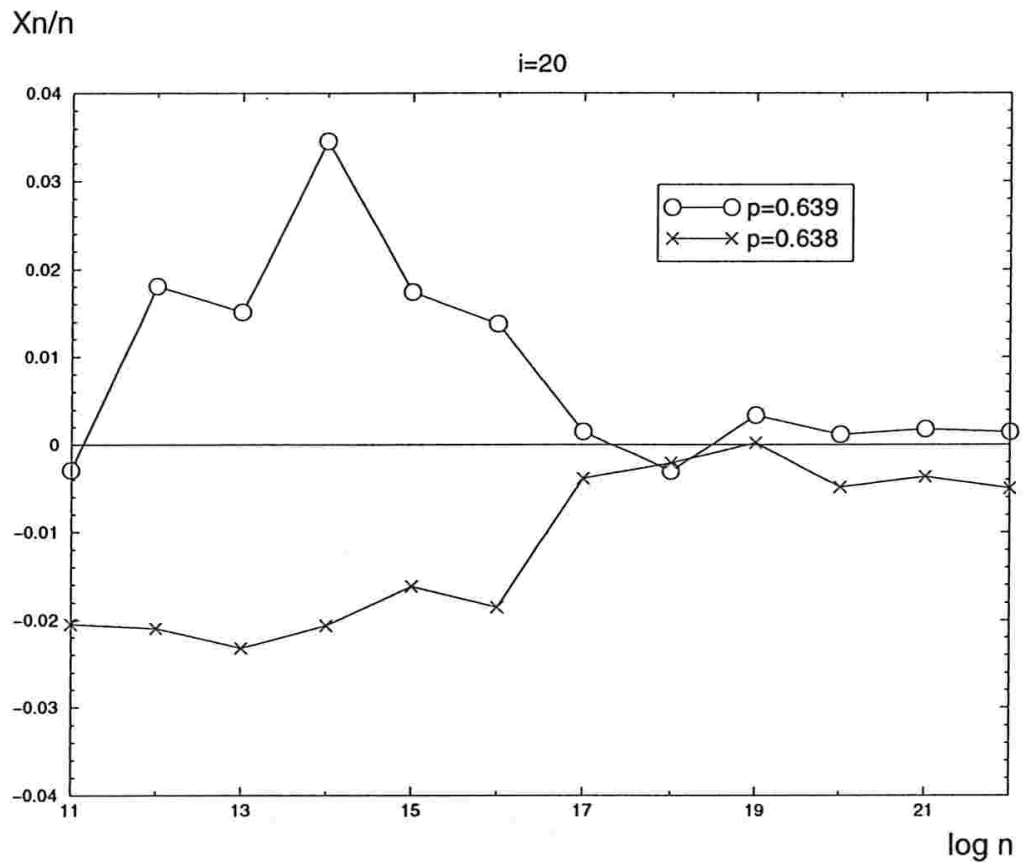

Figura 9: Ampliação e detalhe do gráfico na figura 8 . 
5.1.4 Determinação de $p_{c}^{(40)}$

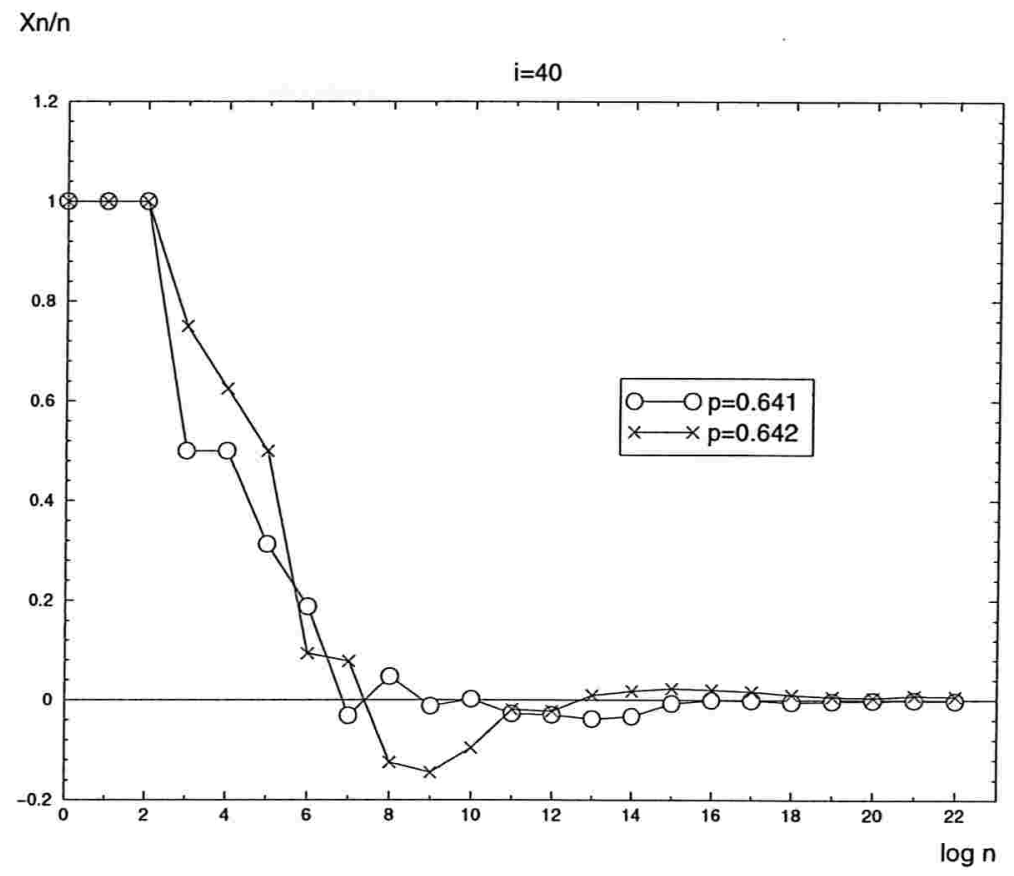

Figura 10: Realizações não acopladas dos processos ${ }_{.641} \bar{X}_{n}^{(40)} / n$ e ${ }_{642} \bar{X}_{n}^{(40)} / n$. 


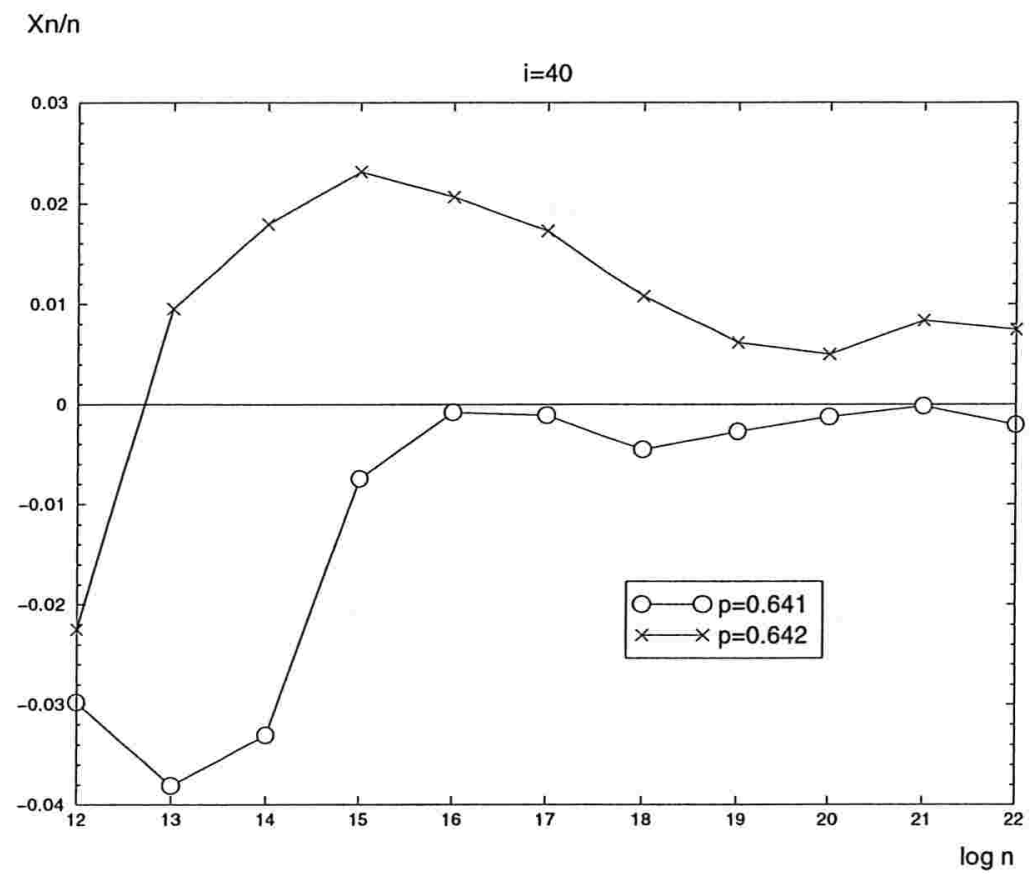

Figura 11: Ampliação e detalhe do gráfico na figura 10 . 
5.1.5 Determinação de $p_{c}^{(100)}$

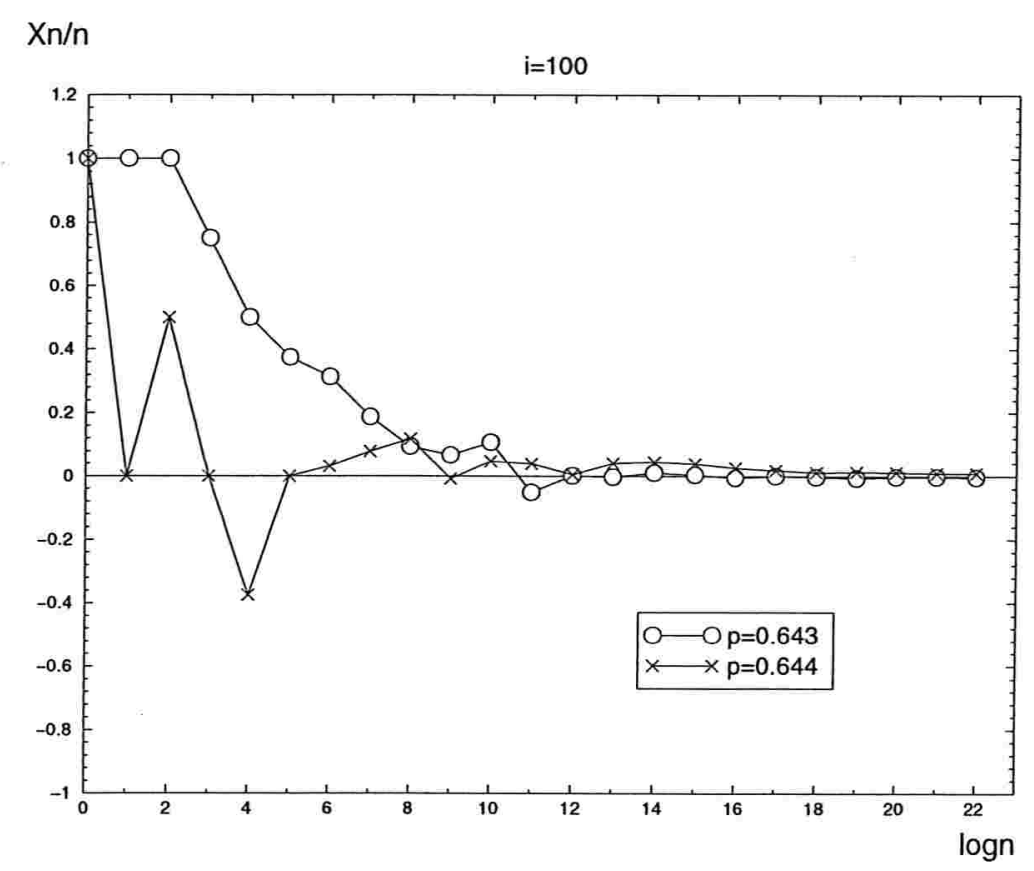

Figura 12: Realizações não acopladas dos processos ${ }_{.643} \bar{X}_{n}^{(100)} / n$ e $.644 \bar{X}_{n}^{(100)} / n$. 


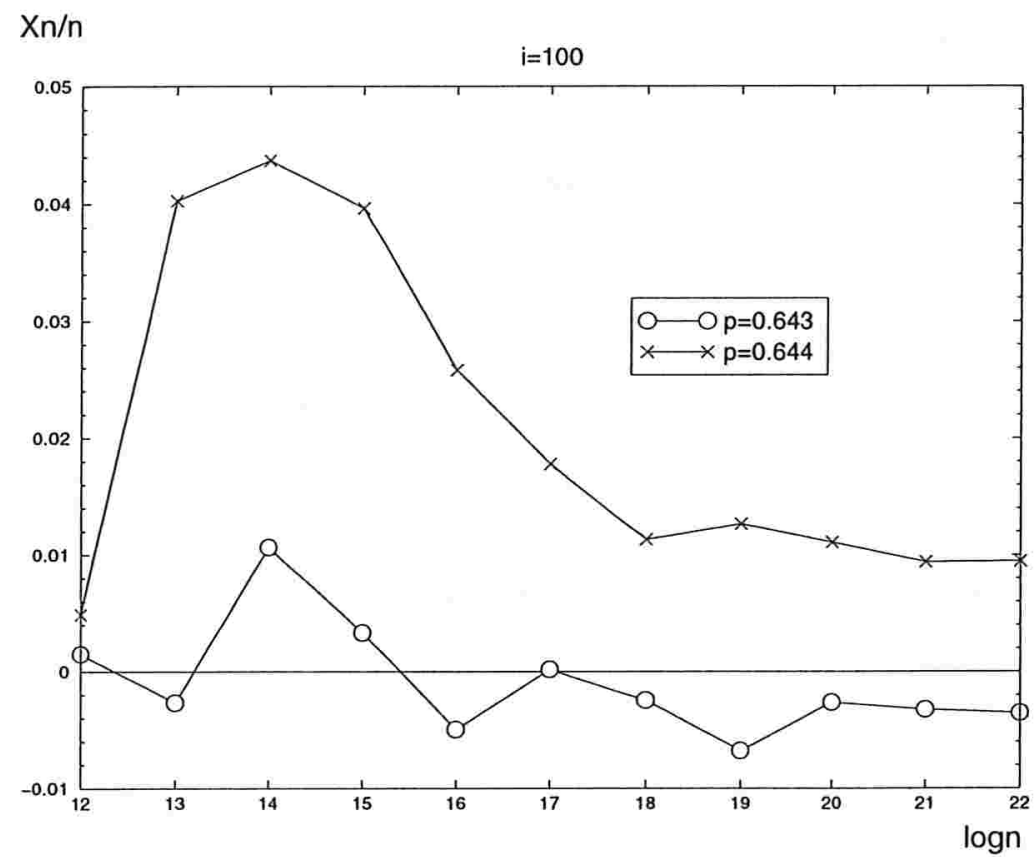

Figura 13: Ampliação e detalhe do gráfico na figura 12. 
5.1.6 Determinação de $p_{c}^{(200)}$

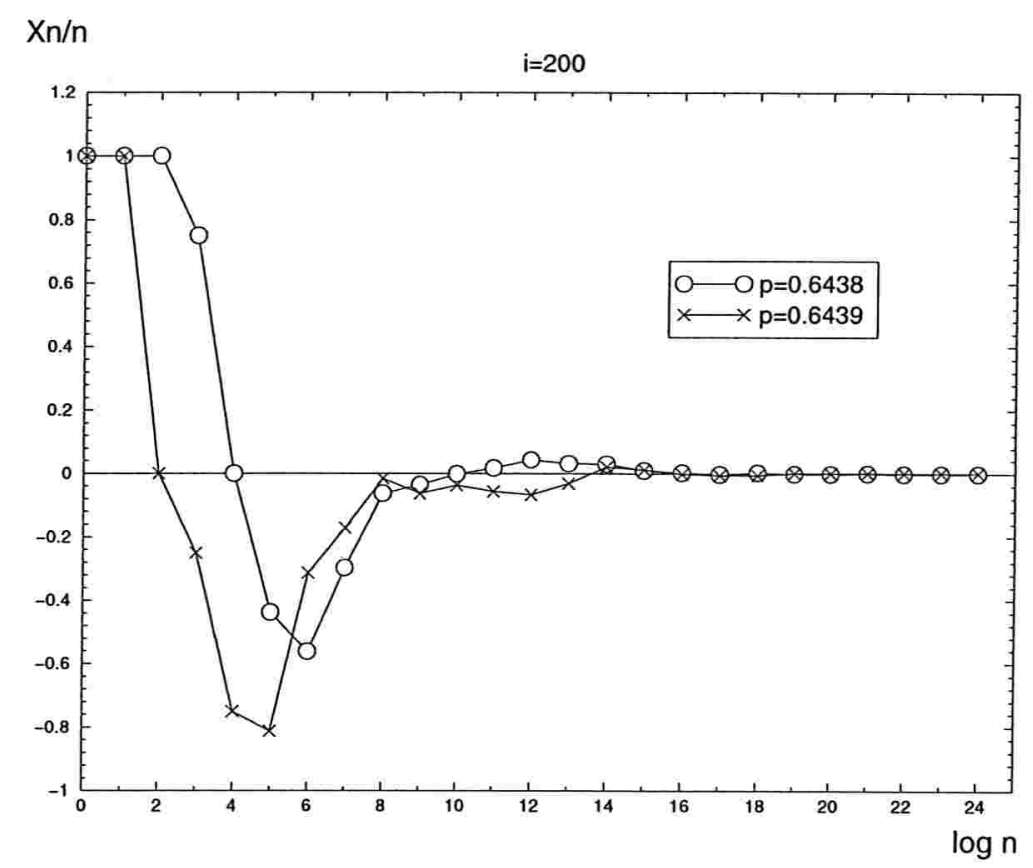

Figura 14: Realizações não acopladas dos processos $.6438 \bar{X}_{n}^{(200)} / n$ e ${ }_{.6439} \bar{X}_{n}^{(200)} / n$. 


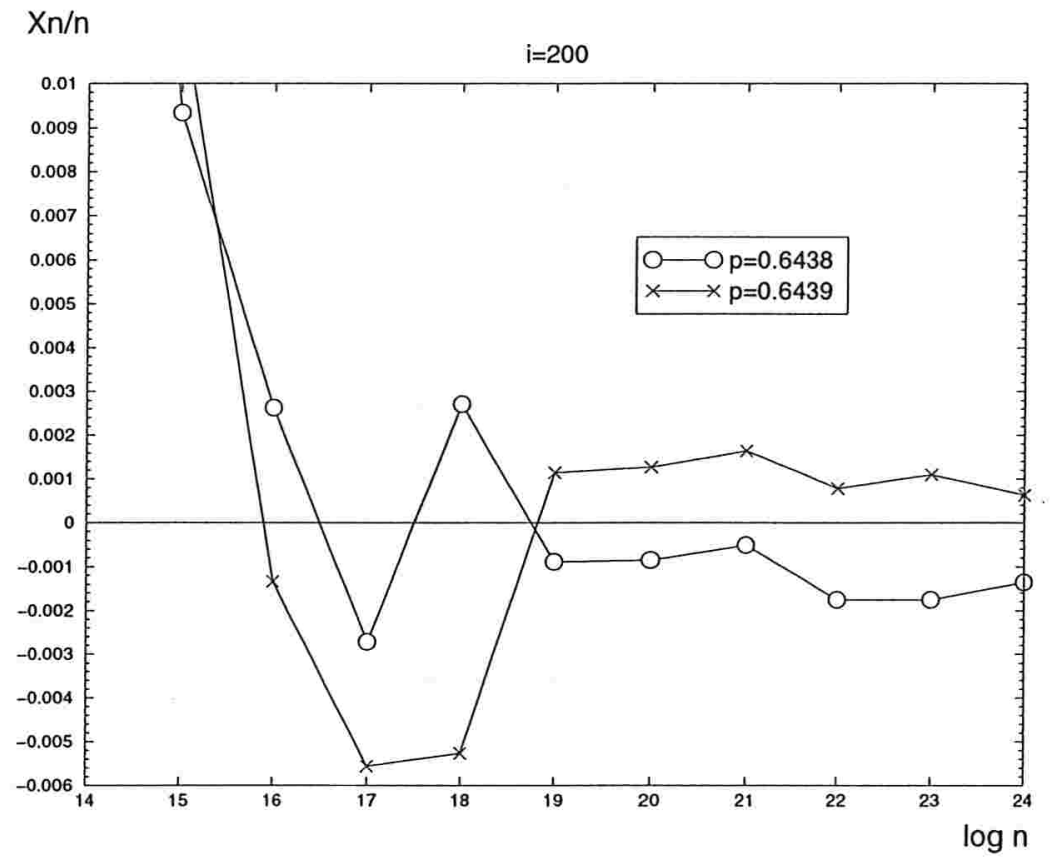

Figura 15: Ampliação e detalhe do gráfico na figura 14 . 
5.1.7 Determinação de $p_{c}^{(1000)}$

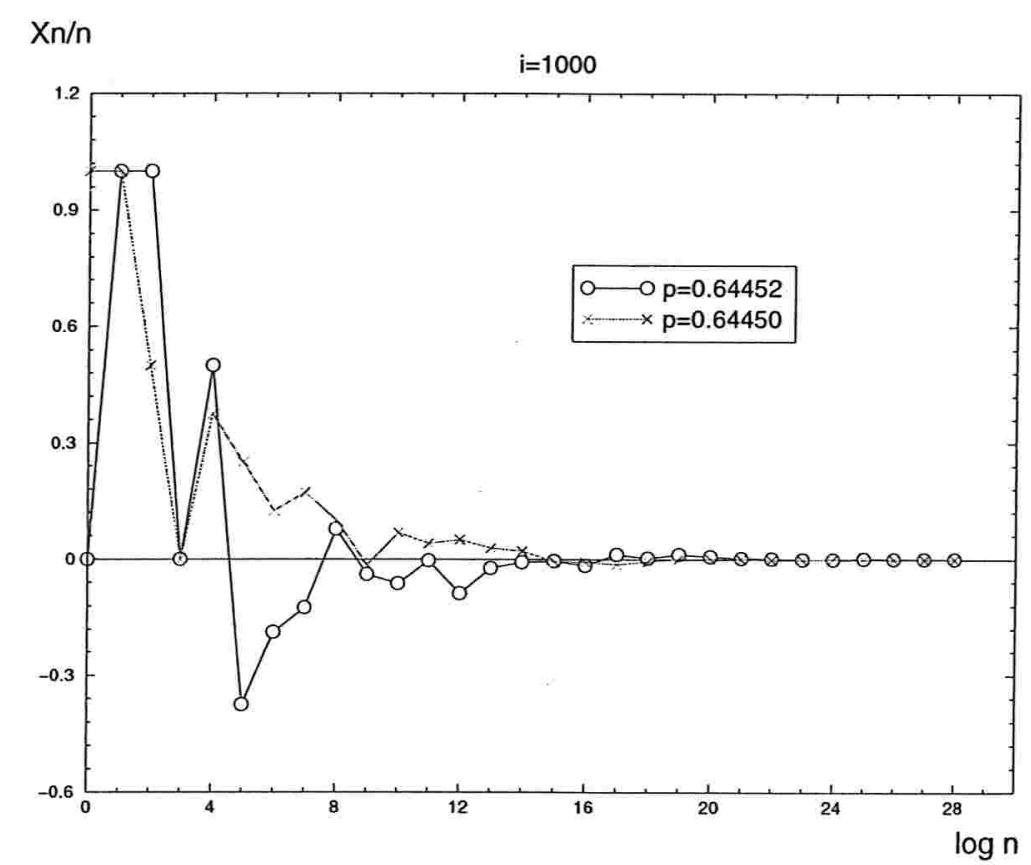

Figura 16: Realizações não acopladas dos processos ${ }_{.64450} \bar{X}_{n}^{(1000)} / n$ e ${ }_{.64452} \bar{X}_{n}^{(1000)} / n$. 


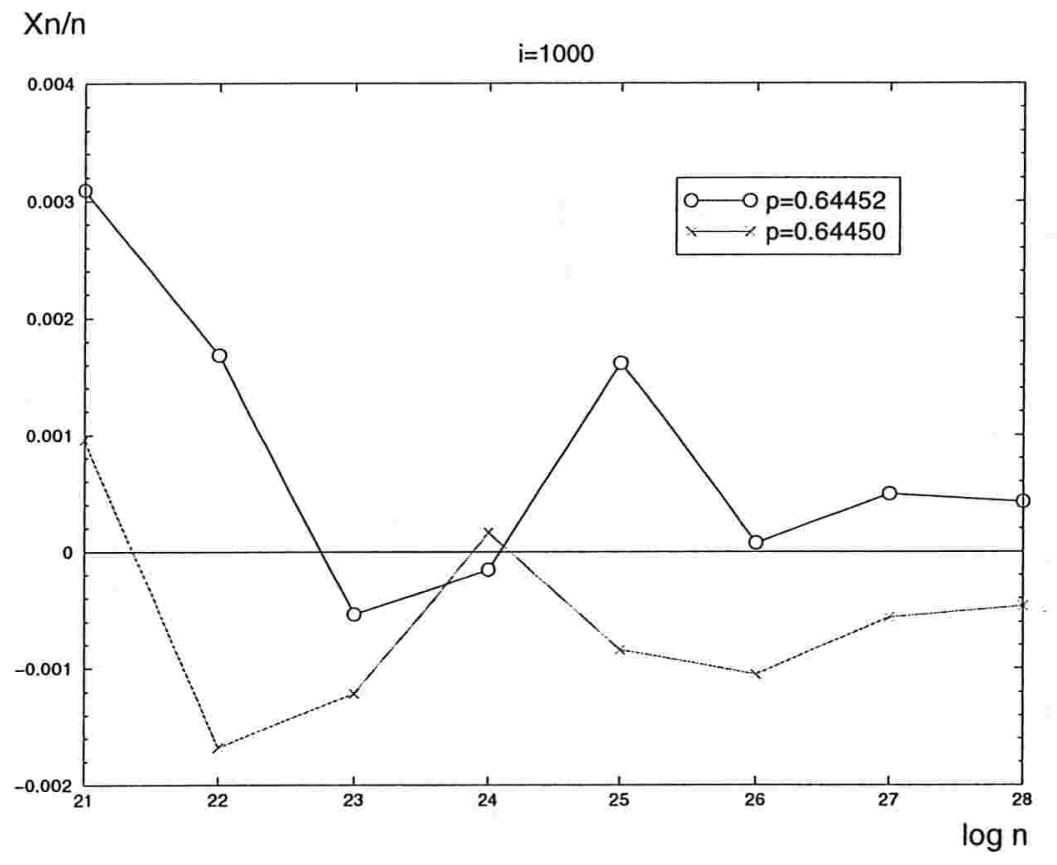

Figura 17: Ampliação e detalhe do gráfico na figura 16 . 


\subsection{Ilustração dos Acoplamentos via Simulação}

Os acoplamentos abaixo foram obtidos construindo-se trajetórias dos diferentes processos estocásticos com a mesma seqüência de números aleatórios:

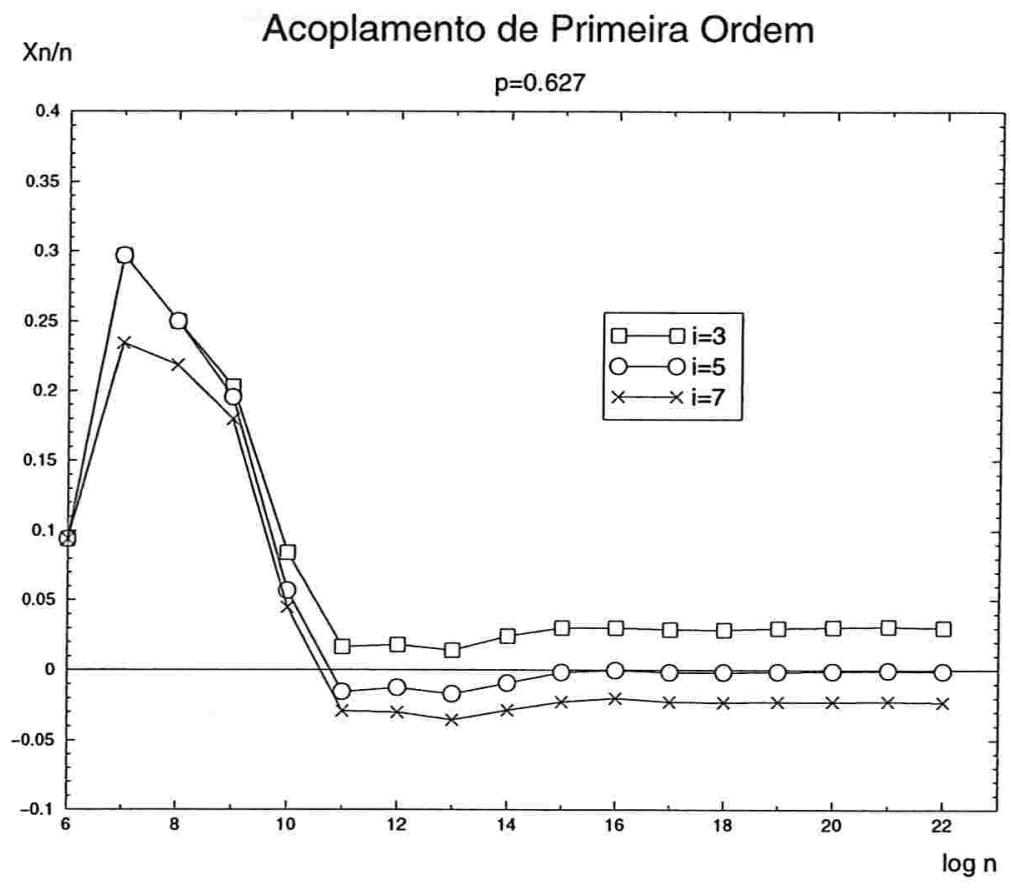

Figura 18: Realizações acopladas de ${ }_{.627} \bar{X}_{n}^{(3)} / n, .627 \bar{X}_{n}^{(5)} / n$, e ${ }_{.627} \bar{X}_{n}^{(7)} / n$. 


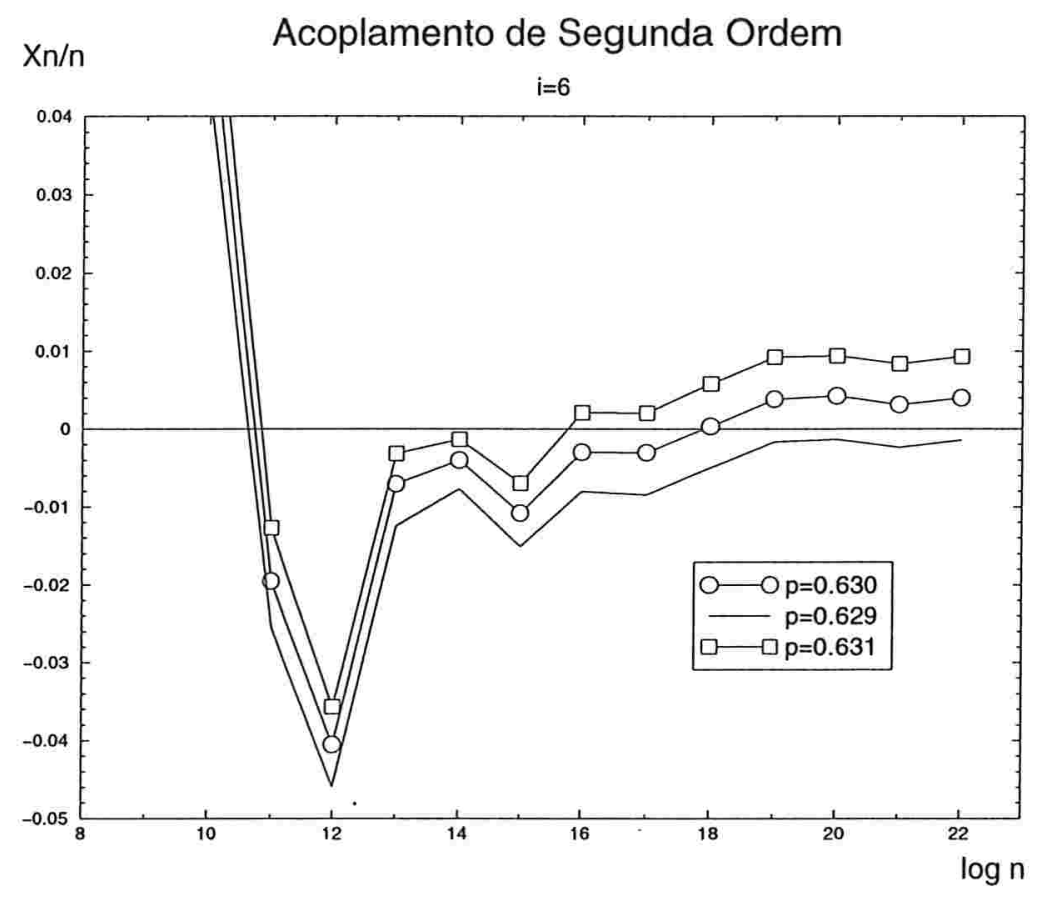

Figura 19: Realizações acopladas de ${ }_{.629} \bar{X}_{n}^{(6)} / n, .630 \bar{X}_{n}^{(6)} / n$, e ${ }_{.631} \bar{X}_{n}^{(6)} / n$. 


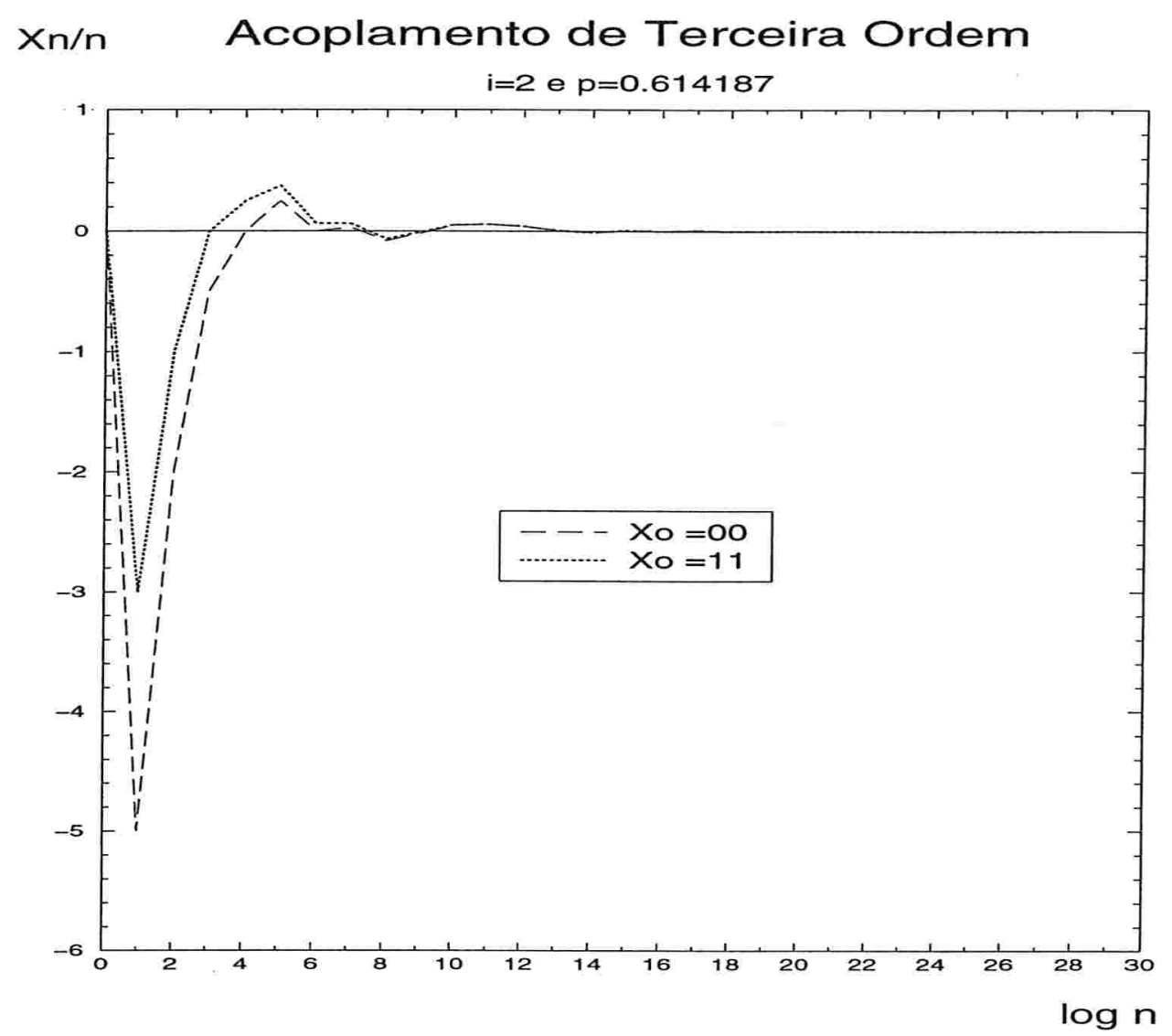

Figura 20: Realizações acopladas de $\underset{p_{c}^{(2)}}{\sigma_{0}} \bar{X}_{n}^{(2)} / n$ e $\underset{p_{c}^{(2)}}{\sigma_{3}} \bar{X}_{n}^{(2)} / n$.

Obs.: $\sigma_{0} \equiv 00, \sigma_{3} \equiv 11$ como na figura 2 . 


\section{A Programa I}

Program MatrizdeTransicao

Integer $\mathrm{N}$

Real*8 P,dP

Parameter $(\mathrm{N}=9, \mathrm{P}=0.63325, \mathrm{dP}=0.00001)$

Integer I,J,K, Confi,Conff,MT,Paux1,Paux2,Paux3,Paux

Integer $\mathrm{P} 0, \mathrm{P} 1, \mathrm{P} 2, \mathrm{P} 3, \mathrm{P} 4, \mathrm{P} 5$

Integer Delta, Cabeca, Grau

Integer MD,IND2

Dimension $\mathrm{MD}\left(0: 2^{* *} \mathrm{~N}-1,0: 4^{*} \mathrm{~N}+6\right)$

Dimension P0 $\left(0: 4^{*} \mathrm{~N}+6\right), \mathrm{P} 1\left(0: 4^{*} \mathrm{~N}+6\right), \mathrm{P} 2\left(0: 4^{*} \mathrm{~N}+6\right)$

Dimension P3 $\left(0: 4^{*} \mathrm{~N}+6\right), \mathrm{P} 4\left(0: 4^{*} \mathrm{~N}+6\right), \mathrm{P} 5\left(0: 4^{*} \mathrm{~N}+6\right)$

Dimension Confi( ( -4$) * \mathrm{~N}-4:+2), \operatorname{Conff}((-4) * \mathrm{~N}-3:+1)$

Dimension MT( $\left.0: 2^{* *} \mathrm{~N}-1,0: 2^{* *} \mathrm{~N}-1,0: 4^{*} \mathrm{~N}+6\right)$

Dimension Paux1(0:4*N+6), Paux $\left(0: 4^{*} N+6\right)$

Dimension Paux2(0:4*N+6),Paux3(0:4*N+6)

Real*8 Q2,SL2,CSL2, MD2,MI2,MAXD,AUXD,X,dX,Y,dY,Z,dZ

Real*8 MDRIFT,EMDRIFT,NRes,NMD,EMD

Dimension SL2 $\left(0: 2^{* *} \mathrm{~N}-1,0: 2^{* *} \mathrm{~N}, 1: 2\right), \mathrm{CSL} 2\left(0: 2^{* *} \mathrm{~N}-1,0: 2^{* *} \mathrm{~N}-1\right)$

Dimension MD2(0:2**N-1,1:2),MI2(0:2**N-1,1:2)

Do $01 \mathrm{I}=0,4 * \mathrm{~N}+6$

$\mathrm{PO}(\mathrm{I})=0$

$\mathrm{P} 1(\mathrm{I})=0$

$\mathrm{P} 2(\mathrm{I})=0$

$\mathrm{P} 3(\mathrm{I})=0$

$\mathrm{P} 4(\mathrm{I})=0$

$\mathrm{P} 5(\mathrm{I})=0$

01 Continue

$\mathrm{P} 1(0)=1$

$\mathrm{P} 2(0)=1$

$\mathrm{P} 3(1)=1$

$\mathrm{P} 4(0)=1$

$\mathrm{P} 5(2)=1$

$\mathrm{P} 2(1)=-1$

$\mathrm{P} 4(2)=-1$

Open(Unit $=1$,File $=$ 'Arq9E.dat')

Do $10 \mathrm{I}=0,2^{* *} \mathrm{~N}-1$

Call Bin0 (I,Confi,N)

Do $20 \mathrm{~J}=0,2^{* *} \mathrm{~N}-1$

c Write $\left(1,{ }^{*}\right)$ 'MT',I,'-',J,'='

Call Bin1 (J,Conff,N)

Call ProbTotal (Confi,Conff,Paux1,N)

Do $22 \mathrm{~K}=0,4^{*} \mathrm{~N}+6$

$\operatorname{MT}(\mathrm{I}, \mathrm{J}, \mathrm{K})=\operatorname{Paux} 1(\mathrm{~K})$

c Write $\left(1,{ }^{*}\right)$ MT $(\mathrm{I}, \mathrm{J}, \mathrm{K})$

22 Continue

c Write $(1, *)$, ,

20 Continue

10 Continue 
$\mathrm{c}=======$ Determinacao Algebrica do Mean Drift $===========$

Do $30 \mathrm{I}=0,2^{* *} \mathrm{~N}-1$

Call Bin0 (I,Confi,N)

Call AtribVet (P0,Paux,N)

Do $40 \mathrm{~J}=1,-2 * \mathrm{~N}-3,-2$

Call AtribVet $(\mathrm{P} 1, \mathrm{Paux} 1, \mathrm{~N})$

Do $50 \mathrm{~K}=1, \mathrm{~J}+2,-2$

GO TO $(51,52,53)$, Confi $(\mathrm{K}-1)+\operatorname{Confi}(\mathrm{K}+1)+1$

51 GO TO 50

52 Call Produto (P3,Paux1,Paux2,N)

Call AtribVet (Paux2,Paux1,N)

GO TO 50

53 Call Produto (P5,Paux1,Paux2,N)

Call AtribVet (Paux2,Paux1,N)

50 Continue

IF( J .NE. $(-2) * \mathrm{~N}-3$ ) THEN

GO TO $(54,55,56)$, Confi( J-1)+Confi $(\mathrm{J}+1)+1$

54 Call AtribVet (P0,Paux1,N)

GO TO 57

55 Call Produto (P2,Paux1,Paux2,N)

Call AtribVet (Paux2,Paux1,N)

GO TO 57

56 Call Produto (P4,Paux1,Paux2,N)

Call AtribVet (Paux2,Paux1,N)

57 Continue

Do $60 \mathrm{~K}=0$, Grau (Paux1,N)

$\operatorname{Paux1}(\mathrm{K})=\operatorname{Paux} 1(\mathrm{~K}) * \mathrm{~J}$

60 Continue

Call Soma (Paux1,Paux,Paux2,N)

Call AtribVet (Paux2,Paux,N)

END IF

40 Continue

Call AtribVet $(\mathrm{P} 4, \mathrm{Paux} 2, \mathrm{~N})$

Do $70 \mathrm{~K}=0,2$

$\operatorname{Paux} 2(\mathrm{~K})=\operatorname{Paux} 2(\mathrm{~K}) *((-2) * \mathrm{~N}-1)$

70 Continue

Paux2(0)=Paux2(0)-2

Call Produto (Paux1,Paux2,Paux3,N)

Call Produto (Paux,P4,Paux2,N)

Call Soma (Paux3,Paux2,Paux,N)

c Write $\left(1,{ }^{*}\right)$ 'MD', $\mathrm{I},{ }^{\prime}=$ '

Do $80 \mathrm{~K}=0,4^{*} \mathrm{~N}+6$

$\operatorname{MD}(\mathrm{I}, \mathrm{K})=\operatorname{Paux}(\mathrm{K})$

c Write $\left(1,{ }^{*}\right) \mathrm{MD}(\mathrm{I}, \mathrm{K})$

80 Continue

30 Continue

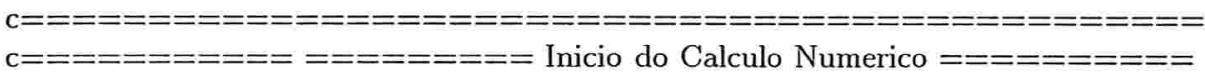

$\mathrm{c}===ニ===$
Write $\left(1,{ }^{*}\right) \cdot \mathrm{I}=', \mathrm{~N}$ 


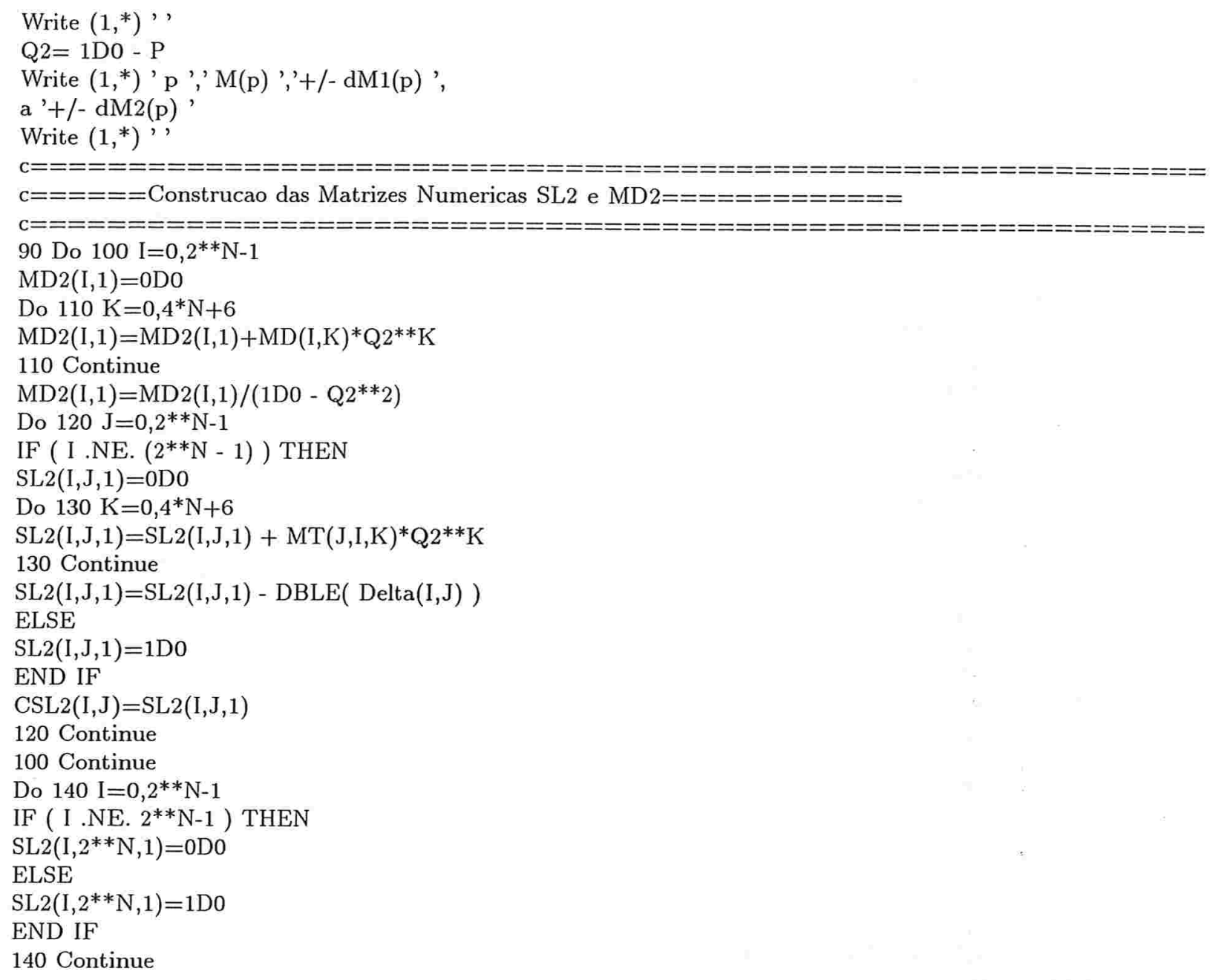

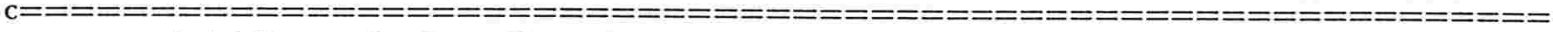
$c====$ Inicializacao dos Erros Numericos $===============0$

$\mathrm{c}=\mathrm{C}=\mathrm{=}=\mathrm{=}=\mathrm{=}=\mathrm{=}=\mathrm{=}=\mathrm{=}=\mathrm{=}=\mathrm{=}=\mathrm{=}=\mathrm{=}=\mathrm{=}=\mathrm{=}=\mathrm{=}=\mathrm{=}=\mathrm{=}=\mathrm{=}=\mathrm{=}=\mathrm{=}=\mathrm{=}=\mathrm{=}=\mathrm{=}=\mathrm{=}$

Do $141 \mathrm{I}=0,2^{* *} \mathrm{~N}-1$

$\operatorname{MD} 2(\mathrm{I}, 2)=\operatorname{DABS}(\operatorname{MD} 2(\mathrm{I}, 1))^{*}(1 \mathrm{D}-15)$

141 Continue

Do $142 \mathrm{I}=0,2^{* *} \mathrm{~N}-2$

Do $143 \mathrm{~J}=0,2^{* *} \mathrm{~N}-1$

$\mathrm{SL} 2(\mathrm{I}, \mathrm{J}, 2)=\operatorname{DABS}(\mathrm{SL} 2(\mathrm{I}, \mathrm{J}, 1)) *(1 \mathrm{D}-15)$

143 Continue

142 Continue

Do $144 \mathrm{~J}=0,2^{* *} \mathrm{~N}$

$\mathrm{SL} 2\left(2^{* * N} \mathrm{~N}-1, \mathrm{~J}, 2\right)=0 \mathrm{D} 0$

144 Continue

Do $145 \mathrm{I}=0,2^{* *} \mathrm{~N}-1$

$\mathrm{SL} 2\left(\mathrm{I}, 2^{* *} \mathrm{~N}, 2\right)=0 \mathrm{D} 0$

145 Continue 


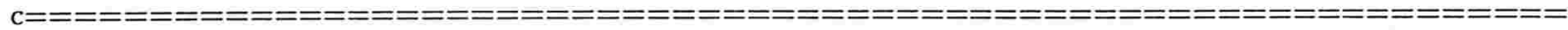
$\mathrm{c}=====$ Escalonamento do Sistema Linear (SL2) $==============$

$\mathrm{c}=\mathrm{=}=\mathrm{=}=\mathrm{=}=\mathrm{=}=\mathrm{=}=\mathrm{=}=\mathrm{=}=\mathrm{=}=\mathrm{=}=\mathrm{=}=\mathrm{=}=\mathrm{=}=\mathrm{=}=\mathrm{=}=\mathrm{=}=\mathrm{=}=\mathrm{=}=\mathrm{=}=\mathrm{=}=\mathrm{=}=\mathrm{=}=\mathrm{=}$

Do $150 \mathrm{I}=0,2^{* *} \mathrm{~N}-2$

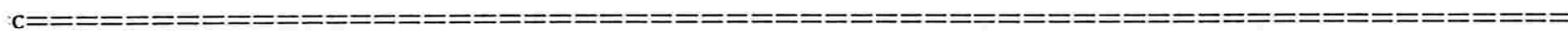

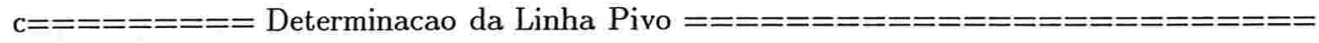

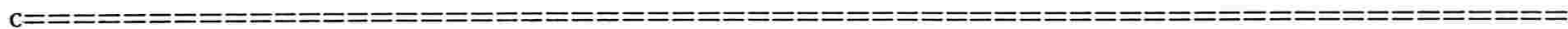
IND2 $=\mathrm{I}$

$\mathrm{MAXD}=0 \mathrm{DO}$

Do $160 \mathrm{~K}=\mathrm{I}, 2 * * \mathrm{~N}-1$

IF ( DABS( SL2(K,I,1) ) .GE. MAXD ) THEN

$\operatorname{MAXD}=\mathrm{DABS}(\operatorname{SL2}(\mathrm{K}, \mathrm{I}, 1))$

$\mathrm{IND} 2=\mathrm{K}$

END IF

160 Continue

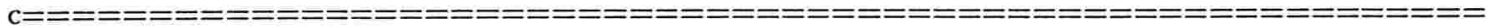

$\mathrm{c}=====$ Troca da Linha I com a Linha IND2 $==============$

$\mathrm{c}=\mathrm{C}=\mathrm{=}=\mathrm{=}=\mathrm{=}=\mathrm{=}=\mathrm{=}=\mathrm{=}=\mathrm{=}=\mathrm{=}=\mathrm{=}=\mathrm{=}=\mathrm{=}=\mathrm{=}=\mathrm{=}=\mathrm{=}=\mathrm{=}=\mathrm{=}=\mathrm{=}=\mathrm{=}=\mathrm{=}=\mathrm{=}$

Do $170 \mathrm{~K}=0,2^{* *} \mathrm{~N}$

$\mathrm{X}=\mathrm{SL} 2(\mathrm{I}, \mathrm{K}, 1)$

$\mathrm{dX}=\mathrm{SL} 2(\mathrm{I}, \mathrm{K}, 2)$

$\mathrm{SL} 2(\mathrm{I}, \mathrm{K}, 1)=\mathrm{SL} 2(\mathrm{IND} 2, \mathrm{~K}, 1)$

$\mathrm{SL} 2(\mathrm{I}, \mathrm{K}, 2)=\mathrm{SL} 2(\mathrm{IND} 2, \mathrm{~K}, 2)$

$\mathrm{SL} 2(\mathrm{IND} 2, \mathrm{~K}, 1)=\mathrm{X}$

$\mathrm{SL} 2(\mathrm{IND} 2, \mathrm{~K}, 2)=\mathrm{dX}$

170 Continue

$\mathrm{c}=\mathrm{=}=\mathrm{=}=\mathrm{=}=\mathrm{=}=\mathrm{=}=\mathrm{=}=\mathrm{=}=\mathrm{=}=\mathrm{=}=\mathrm{=}=\mathrm{=}=\mathrm{=}=\mathrm{=}=\mathrm{=}=\mathrm{=}=\mathrm{=}=\mathrm{=}=\mathrm{=}=\mathrm{=}=\mathrm{=}=\mathrm{=}=\mathrm{=}=\mathrm{=}$

Do $180 \mathrm{~K}=\mathrm{I}+1,2^{* * \mathrm{~N}-1}$

Call DNum(SL2(K,I,1),SL2(K,I,2),SL2(I,I,1),SL2(I,I,2),X,dX)

IF ( dX.LT.-5D-1) THEN

WRITE $\left(1,{ }^{*}\right)$ 'Erro Numerico nao Controlavel'

GO TO 260

END IF

Do $190 \mathrm{~J}=\mathrm{I}+1,2^{* *} \mathrm{~N}$

Call MNum(SL2(I,J,1),SL2(I,J,2),X,dX, Y,dY)

Call SNum(SL2(K,J,1),SL2(K,J,2),-Y,dY,Z,dZ)

$\mathrm{SL} 2(\mathrm{~K}, \mathrm{~J}, 1)=\mathrm{Z}$

$\mathrm{SL} 2(\mathrm{~K}, \mathrm{~J}, 2)=\mathrm{dZ}$

190 Continue

180 Continue

150 Continue

$\mathrm{c}=========$ Fim do Escalonamento do Sist. Linear (SL2) $======$

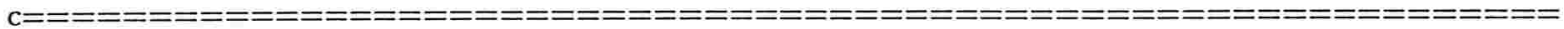
$\mathrm{c}======$ Obtencao da Medida Invarinte $(\mathrm{MI2})============$

$\mathrm{c}=\mathrm{=}=\mathrm{=}=\mathrm{=}=\mathrm{=}=\mathrm{=}=\mathrm{=}=\mathrm{=}=\mathrm{=}=\mathrm{=}=\mathrm{=}=\mathrm{=}=\mathrm{=}=\mathrm{=}=\mathrm{=}=\mathrm{=}=\mathrm{=}=\mathrm{=}=\mathrm{=}=\mathrm{=}=\mathrm{=}=\mathrm{=}=\mathrm{=}$

Do $200 \mathrm{I}=2^{* *} \mathrm{~N}-1,0,-1$

$\mathrm{MI} 2(\mathrm{I}, 1)=\mathrm{SL} 2\left(\mathrm{I}, 2^{* *} \mathrm{~N}, 1\right)$

$\mathrm{MI} 2(\mathrm{I}, 2)=\mathrm{SL} 2\left(\mathrm{I}, 2^{* *} \mathrm{~N}, 2\right)$

Do $210 \mathrm{~J}=\mathrm{I}+1,2^{* *} \mathrm{~N}-1$ 
Call MNum(SL2(I,J,1),SL2(I,J,2),MI2(J,1),MI2(J,2),X,dX)

Call SNum(MI2(I,1),MI2(I,2),-X,dX,Y,dY)

$\mathrm{MI} 2(\mathrm{I}, 1)=\mathrm{Y}$

$\mathrm{MI} 2(\mathrm{I}, 2)=\mathrm{dY}$

210 Continue

Call DNum(MI2(I,1),MI2(I,2),SL2(I,I,1),SL2(I,I,2),X,dX)

IF ( dX.LT.-5D-1 ) THEN

WRITE $\left(1,{ }^{*}\right)$ 'Erro Numerico nao Controlavel'

GO TO 260

END IF

$\mathrm{MI} 2(\mathrm{I}, 1)=\mathrm{X}$

$\mathrm{MI} 2(\mathrm{I}, 2)=\mathrm{dX}$

200 Continue

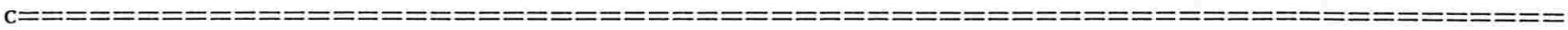
$\mathrm{c}=========$ Obtencao de $\mathrm{M}(\mathrm{p})$ - MDRIFT

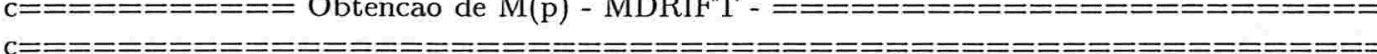

MDRIFT $=0 \mathrm{DO}$

EMDRIFT $=0 \mathrm{DO}$

Do $220 \mathrm{I}=0,2^{* *} \mathrm{~N}-1$

Call MNum(MI2(I,1),MI2(I,2),MD2(I,1),MD2(I,2),X,dX)

Call SNum(X,dX,MDRIFT,EMDRIFT,Y,dY)

MDRIFT $=Y$

$\mathrm{EMDRIFT}=\mathrm{dY}$

220 Continue

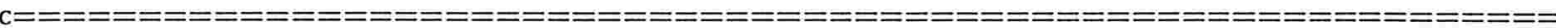

$\mathrm{c}===$ Verificacao do Erro Numerico da Sol.(MI2) do Sist. Linear(SL2) $===$

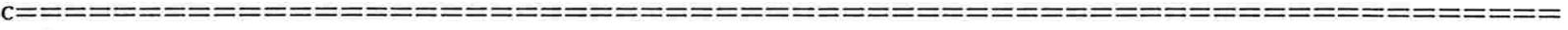

NRes $=0 \mathrm{D} 0$

Do $230 \mathrm{I}=0,2^{* *} \mathrm{~N}-1$

$\mathrm{AUXD}=0 \mathrm{D} 0$

Do $240 \mathrm{~J}=0,2^{* *} \mathrm{~N}-1$

$\mathrm{AUXD}=\mathrm{AUXD}+\mathrm{CSL} 2(\mathrm{I}, \mathrm{J}) * \mathrm{MI} 2(\mathrm{~J}, 1)$

240 Continue

NRes $=$ NRes $+\left(\right.$ DBLE $\left(\operatorname{Delta}\left(I, 2^{* *} \mathrm{~N}-1\right)\right)-$ AUXD $) * * 2$

230 Continue

NRes $=$ DSQRT $($ NRes $)$

$\mathrm{AUXD}=0 \mathrm{D0}$

Do $250 \mathrm{I}=0,2^{* *} \mathrm{~N}-1$

$\mathrm{AUXD}=\mathrm{AUXD}+\mathrm{MD} 2(\mathrm{I}, 1)^{* *} 2$

250 Continue

$\mathrm{NMD}=\mathrm{DSQRT}(\mathrm{AUXD})$

$\mathrm{EMD}=\mathrm{NRes} * \mathrm{NMD}$

$\mathrm{c}=\mathrm{=}=\mathrm{=}=\mathrm{=}=\mathrm{=}=\mathrm{=}=\mathrm{=}=\mathrm{=}=\mathrm{=}=\mathrm{=}=\mathrm{=}=\mathrm{=}=\mathrm{=}=\mathrm{=}=\mathrm{=}=\mathrm{=}=\mathrm{=}=\mathrm{=}=\mathrm{=}=\mathrm{=}=\mathrm{=}=\mathrm{=}=\mathrm{=}=\mathrm{=}=\mathrm{=}=\mathrm{=}=\mathrm{=}$

Write (1,259) (1D0-Q2), (MDRIFT),(EMDRIFT),(EMD)

259 FORMAT (E12.6E2,E16.5E2,E13.5E2,E13.5E2)

$\mathrm{Q} 2=\mathrm{Q} 2-\mathrm{dP}$

IF ( MDRIFT .LE. ODO ) THEN

GO TO 90

END IF

260 Continue

Stop 


\section{End}

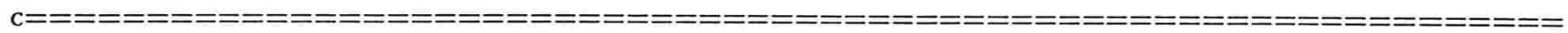

Subroutine Soma $(\mathrm{P} 1, \mathrm{P} 2, \mathrm{~S}, \mathrm{~N})$

Integer P1,P2,S,N

Dimension $\mathrm{P} 1(0: 4 * \mathrm{~N}+6), \mathrm{P} 2(0: 4 * \mathrm{~N}+6), \mathrm{S}(0: 4 * \mathrm{~N}+6)$

Integer I

Do $100 \cdot \mathrm{I}=0,4^{*} \mathrm{~N}+6$

$\mathrm{S}(\mathrm{I})=\mathrm{P} 1(\mathrm{I})+\mathrm{P} 2(\mathrm{I})$

100 Continue

Return

End

$\mathrm{c}=\mathrm{=}=\mathrm{=}=\mathrm{=}=\mathrm{=}=\mathrm{=}=\mathrm{=}=\mathrm{=}=\mathrm{=}=\mathrm{=}=\mathrm{=}=\mathrm{=}=\mathrm{=}=\mathrm{=}=\mathrm{=}=\mathrm{=}=\mathrm{=}=\mathrm{=}=\mathrm{=}=\mathrm{=}=\mathrm{=}=\mathrm{=}=\mathrm{=}=\mathrm{=}=\mathrm{=}=\mathrm{=}$

Subroutine Produto (P1,P2,P,N)

External Grau

Integer $\mathrm{P} 1, \mathrm{P} 2, \mathrm{P}, \mathrm{N}$

Dimension $\mathrm{P} 1\left(0: 4^{*} \mathrm{~N}+6\right), \mathrm{P} 2\left(0: 4^{*} \mathrm{~N}+6\right), \mathrm{P}\left(0: 4^{*} \mathrm{~N}+6\right)$

Integer I,J,Grau

Do $200 \mathrm{I}=0,4^{*} \mathrm{~N}+6$

$\mathrm{P}(\mathrm{I})=0$

200 Continue

Do $210 \mathrm{I}=0, \mathrm{Grau}(\mathrm{P} 1, \mathrm{~N})$

Do $220 \mathrm{~J}=0$, $\mathrm{Grau}(\mathrm{P} 2, \mathrm{~N})$

$\mathrm{P}(\mathrm{I}+\mathrm{J})=\mathrm{P}(\mathrm{I}+\mathrm{J})+\mathrm{P} 1(\mathrm{I}) * \mathrm{P} 2(\mathrm{~J})$

220 Continue

210 Continue

Return

End

$c=============$
Integer Function Grau $(P, N)$

Integer $\mathrm{P}, \mathrm{N}$

Dimension $\mathrm{P}\left(0: 4^{*} \mathrm{~N}+6\right)$

Integer I

$\mathrm{I}=4^{*} \mathrm{~N}+6$

$300 \mathrm{IF}((\mathrm{P}(\mathrm{I})$.EQ. 0) .AND. (I .GT. 0)) THEN

$\mathrm{I}=\mathrm{I}-1$

GO TO 300

END IF

Grau $=$ I

Return

End

$\mathrm{c}==============$
Subroutine Bin0 (Num, Conf, $N$ )

Integer Num, Conf, $\mathrm{N}$

Dimension Conf(-4*N-4:+2)

Integer I,Nb

$\mathrm{Nb}=\mathrm{Num}$

$\operatorname{Conf}(2)=0$

$\operatorname{Conf}(0)=1$

DO $400 \mathrm{I}=0, \mathrm{~N}-1$

$\operatorname{Conf}(0+2 * \mathrm{I}-2 * \mathrm{~N})=\mathrm{MOD}(\mathrm{Nb}, 2)$

$\mathrm{Nb}=\mathrm{Nb} / 2$ 
400 Continue

DO $410 \mathrm{I}=(-2)^{*} \mathrm{~N}-2,(-4) * \mathrm{~N}-4,-2$

$\operatorname{Conf}(\mathrm{I})=1$

410 Continue

Return

End

$\mathrm{c}==============$
Subroutine Bin1 (Num,Conf, $N$ )

Integer Num, Conf, $\mathrm{N}$

Dimension Conf $(-4 * N-3:+1)$

Integer $\mathrm{I}, \mathrm{Nb}$

$\mathrm{Nb}=\mathrm{Num}$

$\operatorname{Conf}(1)=1$

DO $500 \mathrm{I}=0, \mathrm{~N}-1$

$\operatorname{Conf}(1+2 * I-2 * \mathrm{~N})=\operatorname{MOD}(\mathrm{Nb}, 2)$

$\mathrm{Nb}=\mathrm{Nb} / 2$

500 Continue

DO $510 \mathrm{I}=1-2 * \mathrm{~N}-2,(-4) * \mathrm{~N}-3,-2$

$\operatorname{Conf}(\mathrm{I})=1$

510 Continue

Return

End

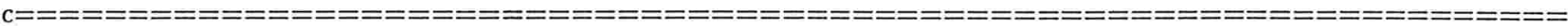

Subroutine AtribVet (P1,P2,N)

Integer P1,P2,N

Dimension P1 $(0: 4 * N+6), \mathrm{P} 2\left(0: 4^{*} \mathrm{~N}+6\right)$

Integer I

DO $550 \mathrm{I}=0,4^{*} \mathrm{~N}+6$

$\mathrm{P} 2(\mathrm{I})=\mathrm{P} 1(\mathrm{I})$

550 Continue

Return

End

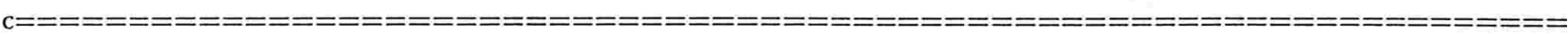

Integer Function Cabeca (Conf,N)

Integer Conf, $\mathrm{N}$

Dimension $\operatorname{Conf}(-4 * \mathrm{~N}-3:+1)$

Integer I

$\mathrm{I}=1$

600 IF ( (Conf(I) .EQ. 0).AND.(I .GT. $-4^{*}$ N-3)) THEN

$\mathrm{I}=\mathrm{I}-2$

GO TO 600

END IF

Cabeca $=\mathrm{I}$

Return

End

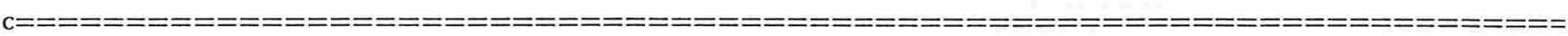

Subroutine Shift (Conf,N)

External Cabeca

Integer Conf, $\mathrm{N}$

Dimension Conf(-4*N-3:+1)

Integer I,Head,Cabeca 


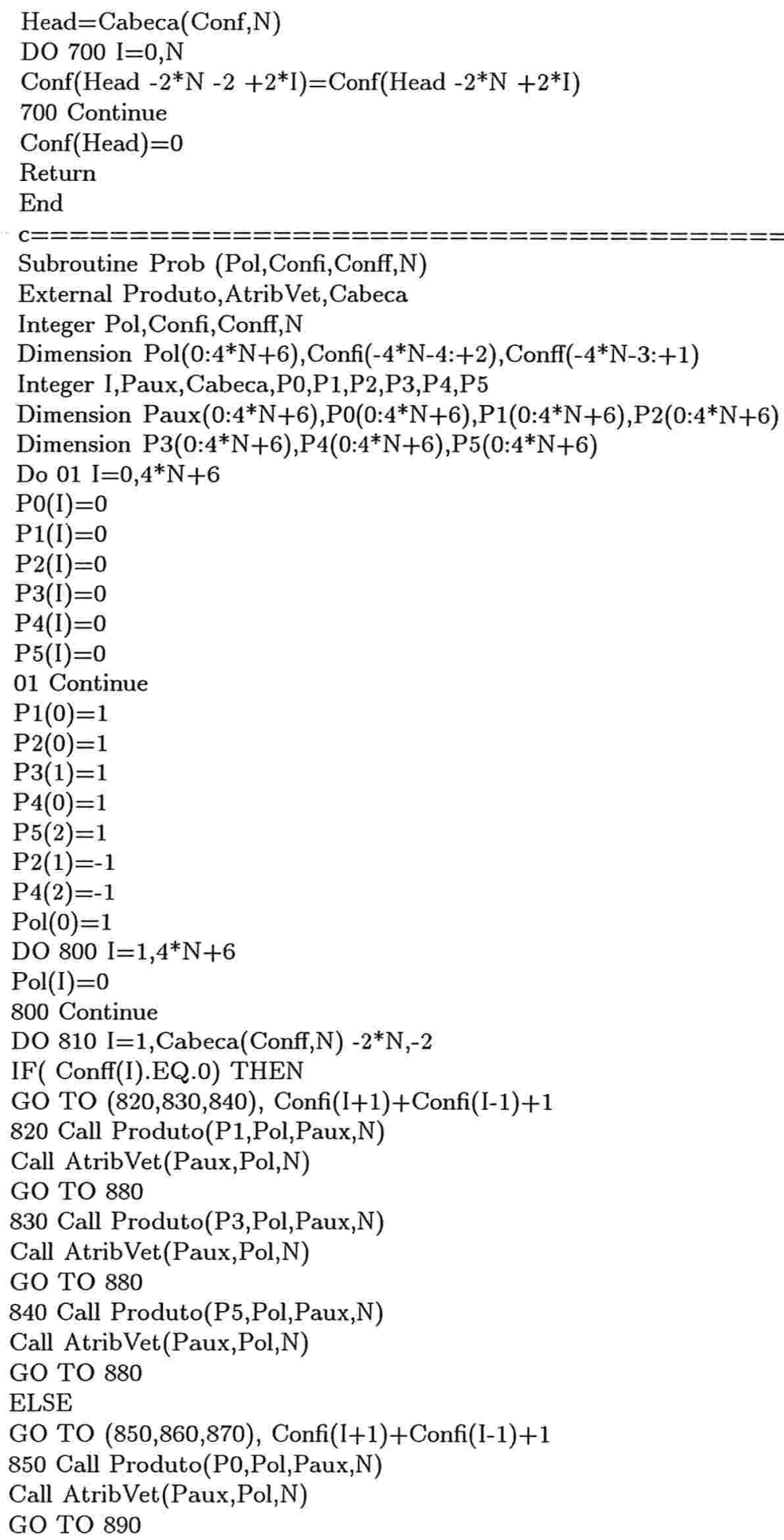


860 Call Produto(P2,Pol,Paux,N)

Call AtribVet(Paux,Pol,N)

GO TO 880

870 Call Produto(P4,Pol,Paux,N)

Call AtribVet(Paux,Pol,N)

GO TO 880

880 END IF

810 Continue

890 Return

End

$\mathrm{c}==================$
Subroutine ProbPG (Pol,Confi,Conff,N)

External Produto,AtribVet, Cabeca

Integer Pol,Conf, Conff, $\mathrm{N}$

Dimension Pol( $\left.0: 4^{*} N+6\right)$, Confi(-4*N-4:+2), Conff(-4*N-3:+1)

Integer I,Paux, Cabeca,P0,P1,P2,P3,P4,P5

Dimension $\mathrm{Paux}\left(0: 4^{*} \mathrm{~N}+6\right), \mathrm{P} 0\left(0: 4^{*} \mathrm{~N}+6\right), \mathrm{P} 1\left(0: 4^{*} \mathrm{~N}+6\right), \mathrm{P} 2\left(0: 4^{*} \mathrm{~N}+6\right)$

Dimension P3(0:4*N+6),P4(0:4*N+6),P5(0:4*N+6)

Do $01 \mathrm{I}=0,4 * \mathrm{~N}+6$

$\mathrm{P} 0(\mathrm{I})=0$

$\mathrm{P} 1(\mathrm{I})=0$

$\mathrm{P} 2(\mathrm{I})=0$

$\mathrm{P} 3(\mathrm{I})=0$

$\mathrm{P} 4(\mathrm{I})=0$

$\mathrm{P} 5(\mathrm{I})=0$

01 Continue

$\mathrm{P} 1(0)=1$

$\mathrm{P} 2(0)=1$

$\mathrm{P} 3(1)=1$

$\mathrm{P} 4(0)=1$

$\mathrm{P} 5(2)=1$

$\mathrm{P} 2(1)=-1$

$\mathrm{P} 4(2)=-1$

$\operatorname{Pol}(0)=1$

DO $900 \mathrm{I}=1,4^{*} \mathrm{~N}+6$

$\operatorname{Pol}(\mathrm{I})=0$

900 Continue

DO $910 \mathrm{I}=1$, Cabeca $($ Conff, $N)+2,-2$

GO TO $(920,930,940)$, Confi $(\mathrm{I}+1)+\operatorname{Confi}(\mathrm{I}-1)+1$

920 Call Produto(P1,Pol,Paux,N)

Call AtribVet(Paux,Pol,N)

GO TO 910

930 Call Produto(P3,Pol,Paux,N)

Call AtribVet(Paux,Pol,N)

GO TO 910

940 Call Produto(P5,Pol,Paux,N)

Call AtribVet(Paux,Pol,N)

GO TO 910

910 Continue

DO $950 \mathrm{I}=$ Cabeca(Conff,N) -2 , Cabeca(Conff,N) $-2 * N,-2$

IF ( Conff(I) .EQ.0 ) THEN 
Call Produto(P5,Pol,Paux,N)

Call AtribVet(Paux,Pol,N)

ELSE

Call Produto(P4,Pol,Paux,N)

Call AtribVet(Paux,Pol,N)

END IF

950 Continue

Return

End

$c=================$
Subroutine ProbTotal(Confi,Conff,Pol,N)

External Prob,Soma,AtribVet,Shift,ProbPG

Integer Confi,Conff,Pol, N

Dimension Confi( $\left(-4^{*} N-4:+2\right), \operatorname{Conff}\left(-4^{*} N-3:+1\right), \operatorname{Pol}\left(0: 4^{*} N+6\right)$

Integer I,Paux1,Paux2

Dimension Paux1(0:4*N+6),Paux2(0:4*N+6)

COMMON/Area1/P0,P1,P2,P3,P4,P5

DO $1000 \mathrm{I}=0,4^{*} \mathrm{~N}+6$

$\operatorname{Pol}(\mathrm{I})=0$

1000 Continue

DO $1010 \mathrm{I}=1,1-2 * \mathrm{~N}-2,-2$

Call Prob(Paux1, Confi,Conff,N)

Call Soma(Pol,Paux1,Paux2,N)

Call AtribVet(Paux2,Pol,N)

Call Shift(Conff,N)

1010 Continue

Call ProbPG(Paux1, Confi,Conff,N)

Call Soma(Pol,Paux1,Paux2,N)

Call AtribVet(Paux2,Pol,N)

Return

End

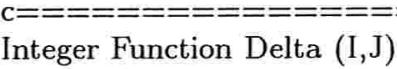

Integer I,J

IF (I.EQ.J) THEN

Delta $=1$

ELSE

Delta $=0$

END IF

Return

End

$\mathrm{c}=\mathrm{=}=\mathrm{=}=\mathrm{=}=\mathrm{=}=\mathrm{=}=\mathrm{=}=\mathrm{=}=\mathrm{=}=\mathrm{=}=\mathrm{=}=\mathrm{=}=\mathrm{=}=\mathrm{=}=\mathrm{=}=\mathrm{=}=\mathrm{=}=\mathrm{=}=\mathrm{=}=\mathrm{=}=\mathrm{=}=\mathrm{=}=\mathrm{=}=\mathrm{=}=\mathrm{=}$

Subroutine SNum (X,dX,Y,dY,Z,dZ)

Real*8 X,dX,Y,dY,Z,dZ

$\mathrm{Z}=\mathrm{X}+\mathrm{Y}$

$\mathrm{dZ}=\mathrm{dX}+\mathrm{dY}$

Return

End

$\mathrm{c}======================$

Real*8 X,dX,Y,dY,Z,dZ 


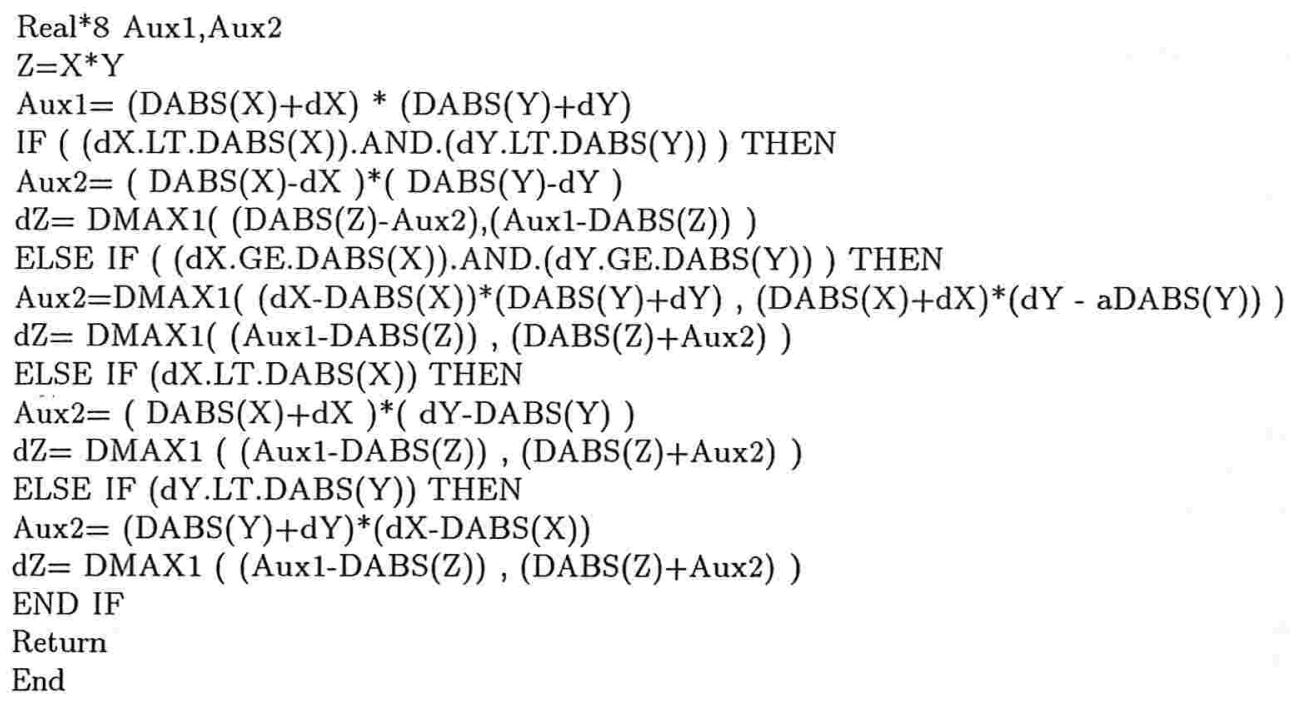




\section{B Programa II}

program percolacaol

integer $\mathrm{m}, \mathrm{n}$, tempo

double precision $\mathrm{p}$

parameter $\left(\mathrm{m}=32, \mathrm{n}=9, \mathrm{p}=0.6333\right.$, tempo $\left.=2^{* *} 30\right)$

logical v1,v2,a,b.

integer $\mathrm{i}, \mathrm{j}, \mathrm{k}$, marc,cont,posicao,ind,idum

real vmd,a1,a2

real ran2

EXTERNAL RAN2

dimension $\mathrm{v} 1(0: \mathrm{m}), \mathrm{v} 2(1: \mathrm{m}+1), \mathrm{vmd}(0: 30)$

OPEN(1,FILE='arqs9df.dat')

idum $=41$

$\mathrm{a}=$.true.

$v 1(0)=$.false.

$\mathrm{v} 2(\mathrm{~m}+1)=$.true.

posicao $=0$

$\operatorname{marc}=0$

do $\mathrm{i}=1, \mathrm{~m}$

$v 1(i)=$.true.

end do

$v 1(3)=$.false.

$\mathrm{v} 1(2)=$.false.

do cont $=1$, tempo

do $\mathrm{j}=1, \mathrm{~m}$

$\mathrm{a} 1=\mathrm{ran} 2$ (idum)

$\mathrm{a} 2=\operatorname{ran} 2$ (idum)

$\mathrm{v} 2(\mathrm{j})=\mathrm{v} 1(\mathrm{j}-1)$.and.(a1.le.p).or.v1(j).and.(a2.le.p)

end do

do $\mathrm{j}=1, \mathrm{~m}+1$

if ( $v 2(j)$.eqv.a) then

go to 20

end if

end do

20 posicao $=$ posicao $+1-(\mathrm{j}-1)^{*} 2$

if (cont.eq. $2^{* *} \operatorname{marc}$ ) then

$\operatorname{vmd}(\operatorname{marc})=\mathrm{real}($ posicao $) / \mathrm{real}$ (cont)

write $\left(1,{ }^{*}\right) \operatorname{marc}, \operatorname{vmd}(\operatorname{marc})$

write $(*, *) \operatorname{marc}, \operatorname{vmd}(\operatorname{marc})$

$\operatorname{marc}=\operatorname{marc}+1$

end if

do $\mathrm{k}=\mathrm{j}, \mathrm{m}$

$\mathrm{v} 1(\mathrm{k}-\mathrm{j}+1)=\mathrm{v} 2(\mathrm{k})$

end do

do $\mathrm{k}=\mathrm{m}-(\mathrm{j}-1)+1, \mathrm{~m}$

$\mathrm{v} 1(\mathrm{k})=$.true.

end do

do $\mathrm{k}=\mathrm{n}+2, \mathrm{~m}$

$\mathrm{v} 1(\mathrm{k})=$.true.

end do

end do 


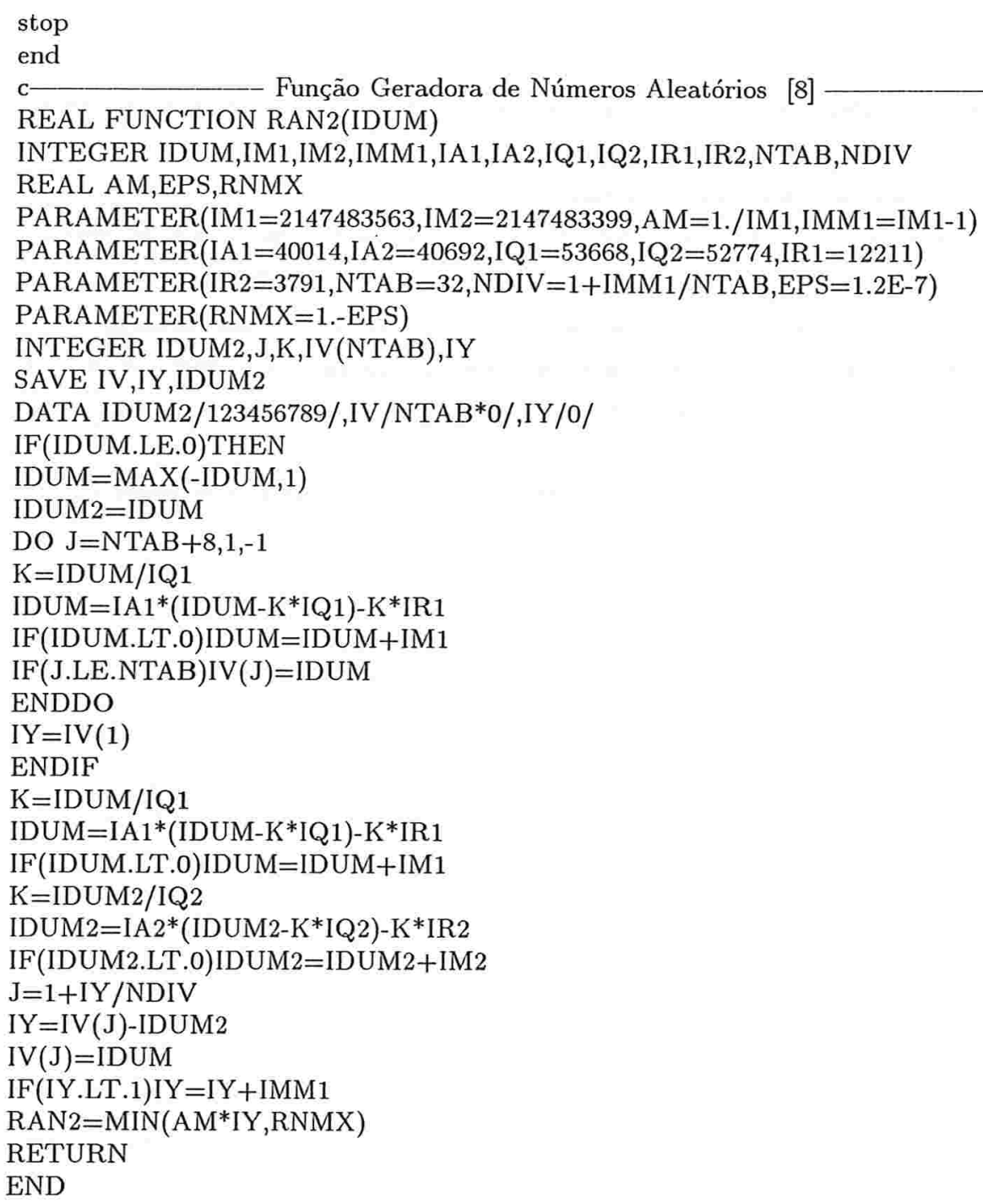




\section{Referências}

[1] R. Durrett. (1984) Oriented Percolation in Two Dimensions. The Annals of Probability, 12(4), 999-1040.

[2] G. Grimmett. (1999) Percolation. Springer Verlag.

[3] G.Fayolle/V.A.Malyshev/M.V.Menshikov. (1995)

Topics in the Constructive Theory of Countable Markov Chains. Cambridge University Press.

[4] Hoel; Port; Stone. (1972) Introduction to Stochastic Processes. Houghton Mifflin Company.

[5] B. R. James. (1996) Probabilidade: Um Curso em Nivel Intermediário. Projeto Euclides.

[6] R. G. Bartle. (1995) The Elements of Integration and Lebesgue Measure. John Willey \& Sons Inc.

[7] J. H. Wilkinson. (1963) Rounding Errors in Algebraic Processes. Her Majesty's Stationary Office.

[8] W. H. Press. (1992)

Numerical Recipes in FORTRAN: the art of scientific computing. Cambridge University Press. 